\title{
HAWK-I infrared supernova search in starburst galaxies *
}

\author{
M. Miluzio ${ }^{1}$, E. Cappellaro ${ }^{2}$, M. T. Botticella ${ }^{3}$, G. Cresci ${ }^{4}$, L. Greggio ${ }^{2}$, F. Mannucci ${ }^{4}$, S. Benetti ${ }^{2}$, F. Bufano ${ }^{5}$, \\ N. Elias-Rosa ${ }^{6}$, A. Pastorello ${ }^{2}$, M. Turatto ${ }^{2}$, and L. Zampieri ${ }^{2}$ \\ ${ }^{1}$ Department of Astronomy, Padova University, Vicolo dell'Osservatorio 3, 35122 Padova, Italy \\ e-mail: matteo.miluzio@unipd.it \\ 2 INAF, Osservatorio Astronomico di Padova, vicolo dell'Osservatorio 5, 35122 Padova, Italy \\ 3 INAF, Osservatorio Astronomico di Capodimonte, Salita Moiariello 16, 80131 Napoli, Italy \\ 4 INAF, Osservatorio Astrofisico di Arcetri, Largo Enrico Fermi 5, 50125 Firenze, Italy \\ 5 Departamento de Ciencias Fisicas, Universidad Andrés Bello, Av. República 252, 8320000 Santiago, Chile \\ ${ }^{6}$ Institut de Ciéncies de l'Espai (IEEC-CSIC), Facultat de Ciéncies, Campus UAB, 08193 Bellaterra, Spain
}

Received 30 January 2013 / Accepted 14 March 2013

\section{ABSTRACT}

\begin{abstract}
Context. The use of SN rates to probe explosion scenarios and to trace the cosmic star formation history received a boost from a number of synoptic surveys. There has been a recent claim of a mismatch by a factor of two between star formation and core collapse $\mathrm{SN}$ rates, and different explanations have been proposed for this discrepancy.

Aims. We attempted an independent test of the relation between star formation and supernova rates in the extreme environment of starburst galaxies, where both star formation and extinction are extremely high.

Methods. To this aim we conducted an infrared supernova search in a sample of local starbursts galaxies. The rationale behind searching in the infrared is to reduce the bias due to extinction, which is one of the putative reasons for the observed discrepancy between star formation and supernova rates. To evaluate the outcome of the search we developed a MonteCarlo simulation tool that is used to predict the number and properties of the expected supernovae based on the search characteristics and the current understanding of starburst galaxies and supernovae.

Results. During the search we discovered 6 supernovae (4 with spectroscopic classification), which is in excellent agreement with the prediction of the MonteCarlo simulation tool that is, on average, $5.3 \pm 2.3$ events.

Conclusions. The number of supernovae detected in starburst galaxies is consistent with what is predicted from their high star formation rate when we recognize that a major fraction $(\sim 60 \%)$ of the events remain hidden in the inaccessible, high-density nuclear regions because of a combination of reduced search efficiency and high extinction.
\end{abstract}

Key words. supernovae: general - galaxies: starburst - galaxies: star formation - infrared: galaxies - infrared: stars

\section{Introduction}

The rate of supernovae ( $\mathrm{SNe}$ ) is a key quantity in astrophysics that provides a crucial test for stellar evolution theory and input for the modeling of galaxy evolution with a direct impact on the chemical enrichment and the feedback mechanism. Because of their short-lived progenitors, core-collapse SNe (SN CC) trace the current star formation rate (SFR). Conversely, for an adopted SFR, measurements of the SN CC rates give information on the mass range of their progenitors, as well as on the slope of the initial mass function at the high-mass end. Resulting from the thermonuclear explosion of a white dwarf in a binary system, SN Ia show a wide range of delay times from star formation to explosion. Therefore, the SN Ia rate reflects the long-term star formation history of the parent stellar system. Recently, it has been claimed that a significant fraction of SN Ia have a short delay time, possibly as short as $10^{7}$ years (Mannucci et al. 2006). As for CC SN, the rate of such prompt SN Ia events is expected to be proportional to the current SFR.

In one of the early attempts to compare the $\mathrm{SN}$ and $\mathrm{SF}$ rates, Cappellaro et al. (1999) found that the SN CC rate in galaxies with different $U-V$ color matches the predicted SFR when

* ESO proposal: 083.D-0259, 085.D-0335, 085.D-0348, 087.D0494, 087.D-0922. GTC proposal: GTC50-11B. adopting a mass range $10 M_{\odot}<M<40 M_{\odot}$ for the SN CC progenitors. In the last decade there was enormous improvement in the measurement of the cosmic SFR with the careful combination of many different probes (eg. Hopkins \& Beacom 2006). A most relevant feature is that the SFR reaches a maximum at a redshift $z \sim 1$ and hereafter begins to decrease to the current rate, which is over one order of magnitude lower than at peak.

A significant effort was also devoted to the measurement of the cosmic SN rate: although much of the focus was for type SN Ia, a few estimates of the SN CC rates have also been published both for the local Universe (Li et al. 2011a) and at high redshifts (Dahlen et al. 2004, 2012; Cappellaro et al. 2005; Botticella et al. 2008; Bazin et al. 2009; Graur et al. 2011; Melinder et al. 2012). While the new local SN CC rate confirms previous results, with much higher statistics and lower systematic errors, the evolution with redshift was found to track the SFR evolution very well, considering the large uncertainties in the extinction corrections. Again, to best match the observed SN and SF rates, it was argued that the lower limit for SN CC progenitor had to be $\sim 10 M_{\odot}$ (Botticella et al. 2008; Blanc \& Greggio 2008).

At about the same time, following a different line of research, the analysis of archival images allowed the identification of the precursors for a number of nearby SN CC. From the often very 
scanty but valuable photometry, and using stellar evolution models, one can estimate the SN precursor mass. The uncertainties are in general quite large, as confirmed from the discrepancy in the mass estimates from different groups, but this analysis suggests a lower limit for SN CC progenitors of $8 \pm 1 M_{\odot}$ (Smartt 2009). If this value is adopted, the observed $S N$ rates would be a factor two lower than those expected from the observed SFR. This was identified by some authors as an "SN rate problem" (e.g. Horiuchi et al. 2011). While one should remind that the uncertainties on SFR rate calibrations are still large (Botticella et al. 2012; Kennicutt \& Evans 2012), it also true that there is a number of possible biases in the SN rate estimates. The two most severe are the possible underestimation of a large population of faint SN CC and/or the underestimation of the correction for extinction (Horiuchi et al. 2011; Mattila et al. 2012).

In particular, Mannucci et al. (2007), Cresci et al. (2007), and more recently, Mattila et al. (2012) argue that a significant fraction of SN CC remains hidden in the nuclear region of starburst galaxies, with a loss of up to $\sim 70-90 \%$ in the highly dustenshrouded environments of (ultra-)luminous infrared galaxies(U/LIRGs). This effect is expected to be more important at high redshift because of the larger fraction of starburst galaxies. Indeed, when a correction for this hidden $\mathrm{SN}$ fraction is included in the rate calculation the discrepancy between SN and SF rates at high redshifts seems to disappear (Melinder et al. 2012; Dahlen et al. 2012; the "missing fraction" correction adopted in these works was from Mattila et al. 2012). It is currently unclear if this effect is large enough to also explain the discrepancy observed in the local Universe with somewhat conflicting evidence from the statistics of SNe in the Local Group galaxies (Botticella et al. 2012; Mattila et al. 2012) and largesample SN searches (Li et al. 2011a).

Entering in this debate, we planned for an infrared SN search in a sample of local starburst galaxies (SBs). The idea was to verify the link between SN and SF rates in an environment where star formation is very high, one to two orders of magnitude higher than in normal star-forming galaxies. By observing in the $K$-band we were aiming to reduce the bias due to extinction $\left(A_{K} \sim 0.1 A_{V}\right)$.

The idea is not new. A first attempt at a dedicated SN search in SBs was performed in the optical band by Richmond et al. (1998). During the search only a handful of events were detected, leading the authors to conclude that the rate of (unobscured) $\mathrm{SNe}$ in SBs is the same as in quiescent galaxies. A similar conclusion was reached by Navasardyan et al. (2001), again based on optical data. As for infrared SN search, after a few unsuccessful attempts (Grossan et al. 1999; Bregman et al. 2000), the first results of a systematic search in SBs were reported by Maiolino et al. (2002) and Mannucci et al. (2003). They find that the observed SN rate in SBs was indeed one order of magnitude higher then expected for the galaxy blue luminosities but still three to ten times lower than would be expected from the far infrared (FIR) luminosity. Among the possible explanations for the remaining discrepancy, they suggested extreme extinction in the galaxy nuclear regions $\left(A_{V}>25 \mathrm{mag}\right)$, which would $\operatorname{dim} \mathrm{SNe}$ even in the near-IR, and insufficient spatial resolution to probe the very nuclear regions. The reliability of the use of an NIR search for obscured $\mathrm{SNe}$ in the nuclear and circumnuclear regions of active starburst galaxies was also investigated by Mattila $\&$ Meikle (2001) in particular taking the problem of extinction into account. They conclude that with a modest investment of observational time it may be possible to discover a number of nuclear SNe. A negative search for transients in NICMOS images retrieved from the Hubble Space Telescope archive suggests that the same biases probably also affect space-based, high spatial resolution observations (Cresci et al. 2007).

The same approach was used by Mattila et al. (2007b) but with ground-based, adaptive optics (AO) assisted observations. The application of this technique led to the discovery of a handful of SNe (Kankare et al. 2008, 2012) but not yet to an estimate of the SN CC rate.

Until now, about a dozen SNe have been discovered by IR $\mathrm{SN}$ searches, not all with spectroscopic confirmation. The number is higher if we include also events first detected in the optical and rediscovered by the IR searches. Therefore the statistics are still very low and many of the original questions are still unanswered. This gave us the motivations to make a new attempt exploiting the opportunity offered by HAWK-I, the infrared camera mounted at the ESO VLT telescope.

The paper is divided into two parts. The first part describes the observing program, namely the galaxy sample and the search strategy in Sect. 2.1, the data reduction in Sect. 2.3, the SN discoveries and classification in Sect. 2.4, while in Sect. 2.5 we explain the procedure to estimate the search detection efficiency. The second part is devoted to the description of a simulation tool that is used to predict the number of expected SN detections (Sect. 3), based on our current knowledge of SBs properties and on the specific features of our SN search. Finally, we compare the number and properties of the expected and observed events (Sect. 4) and draw our conclusions (Sect. 5).

Throughout this paper we assume the following cosmological parameters: $H_{0}=72 \mathrm{~km} \mathrm{~s}^{-1} \mathrm{Mpc}^{-1}, \Omega_{\Lambda}=0.73$ and $\Omega_{\mathrm{M}}=$ 0.27 .

\section{The $\mathrm{SN}$ search program}

\subsection{Galaxy sample}

Starbursts are galaxies with very high SFR in the range of 10-100 $M_{\odot} \mathrm{yr}^{-1}$ compared to the few $M_{\odot} \mathrm{yr}^{-1}$ of normal starforming galaxies in the local universe. Given that in a typical galaxy the very high SFR will rapidly consume the gas reservoir, it is thought that the starburst is a temporary phase in the galaxy evolution. That many SBs are in close pairs or have disturbed morphologies points to the interaction as a dominant, although possibly not unique, reason behind the phenomena (Gallagher 1993). The ultraviolet radiation from young, massive stars heats the surrounding dust and is re-emitted in the far infrared. Indeed the most luminous SBs in the local Universe are LIRGs with $11<\log \left(L_{\mathrm{IR}} / L_{\odot}\right)<12$ and ULIRGs with $\log \left(L_{\mathrm{IR}} / L_{\odot}\right)>12$ (Sanders \& Mirabel 1996).

For our project we selected a sample of SBs with total infrared (TIR) luminosity $\log \left(L_{\mathrm{TIR}} / L_{\odot}\right)>11$ and redshift $z<0.07$ from the IRAS Revised Bright Galaxy Sample (Sanders et al. 2003). With the additional requirement that the targets are accessible from Paranal in the April to September observing season (to fit in one of the ESO allocation period) we retrieved a sample of $30 \mathrm{SBs}$.

The list of SBs is reported in Table 1. Along with the galaxy name and equatorial coordinates (Cols. 1-3) we report the heliocentric redshift (Col. 4), $\log L_{\mathrm{TIR}}$ and $\log L_{B}$ (Cols. 5 and 6; cf. Sect. 3.1.1), the Hubble type (Col. 7), the SFR, and the expected SN rates (Cols. 8, 9) derived from $L_{\mathrm{TIR}}$ as described in Sect. 3.1.1. Galaxy data have been retrieved from $\mathrm{NED}^{1}$. In the

1 The NASA/IPAC Extragalactic Database (NED) is operated by the Jet Propulsion Laboratory, California Institute of Technology, under contract with the National Aeronautics and Space Administration. 
Table 1. The SB galaxy sample.

\begin{tabular}{|c|c|c|c|c|c|c|c|c|c|}
\hline $\begin{array}{l}\text { Galaxy } \\
\text { designation }\end{array}$ & \multicolumn{2}{|c|}{$\mathrm{J} 2000.0$} & Redshift & $\begin{array}{c}\log L_{\mathrm{TIR}} \\
{\left[L_{\odot}\right]}\end{array}$ & $\begin{array}{c}\log L_{B} \\
{\left[L_{\odot}\right]}\end{array}$ & $\begin{array}{c}\text { Hubble } \\
\text { type }\end{array}$ & $\begin{array}{c}\mathrm{SFR} \\
{\left[M_{\odot} \mathrm{yr}^{-1}\right]}\end{array}$ & $\begin{array}{c}\text { SN rate } \\
{\left[\mathrm{SN} \mathrm{yr}^{-1}\right]}\end{array}$ & $\mathrm{SNe}$ \\
\hline CGCG011-076 & 112113.3 & -025908 & 0.025 & 11.28 & 10.35 & 2.9 & 32.1 & 0.38 & \\
\hline CGCG043-099 & 130149.9 & +042001 & 0.037 & 11.59 & 10.51 & 3.4 & 65.4 & 0.77 & \\
\hline ESO148-IG002 & 231546.6 & -590314 & 0.045 & 11.94 & 10.82 & 7.9 & 148.8 & 1.75 & \\
\hline ESO239-IG002 & 224939.6 & -485101 & 0.043 & 11.75 & 10.88 & -0.1 & 95.7 & 1.13 & \\
\hline ESO244-G012 & 011808.6 & -442740 & 0.023 & 11.32 & 10.20 & 5.3 & 35.7 & 0.42 & \\
\hline ESO264-G036 & 104307.0 & -461243 & 0.023 & 11.24 & 10.70 & 1.5 & 29.1 & 0.34 & \\
\hline ESO286-IG019 & 205827.4 & -423857 & 0.043 & 11.95 & 11.13 & 10.0 & 151.6 & 1.78 & \\
\hline ESO440-IG058 & 120653.0 & -315708 & 0.023 & 11.33 & 10.17 & 99.0 & 36.3 & 0.43 & \\
\hline ESO507-G070 & 130251.3 & -235510 & 0.021 & 11.44 & 10.67 & 6.8 & 46.6 & 0.55 & \\
\hline IC 1623A/B & 010746.3 & -173032 & 0.020 & 11.63 & 10.42 & 6.0 & 72.7 & 0.86 & PSN 2011 \\
\hline IC 2545 & 100604.2 & -335304 & 0.034 & 11.66 & 10.58 & -0.1 & 77.7 & 0.92 & \\
\hline IC 2810 & 112547.3 & +144023 & 0.034 & 11.60 & 10.61 & 1.5 & 68.0 & 0.80 & \\
\hline IC $4687 / 6$ & 181338.6 & -574336 & 0.017 & 11.45 & 10.43 & 2.9 & 47.3 & 0.56 & PSN 2010 \\
\hline IRAS12224-0624 & 122502.8 & -064044 & 0.026 & 11.30 & 9.83 & 2.9 & 34.0 & 0.40 & \\
\hline IRAS14378-3651 & 144057.8 & -370425 & 0.068 & 12.13 & 10.40 & 5.1 & 233.0 & 2.74 & \\
\hline IRAS16399-0937 & 164239.2 & -094311 & 0.027 & 11.55 & 10.39 & 10.0 & 60.3 & 0.71 & \\
\hline IRAS17207-0014 & 172321.4 & -001700 & 0.043 & 12.42 & 10.32 & -50 & 447.5 & 5.27 & \\
\hline IRAS18090+0130 & 181137.3 & +013140 & 0.029 & 11.63 & 10.54 & 2.0 & 71.7 & 0.84 & \\
\hline MCG-02-01-051/2 & 001851.4 & -102233 & 0.027 & 11.41 & 10.59 & 3.1 & 44.4 & 0.52 & 2010 hp \\
\hline MCG-03-04-014 & 011008.5 & -165114 & 0.035 & 11.59 & 10.53 & -5.0 & 66.1 & 0.78 & \\
\hline NGC 0034 & 001106.6 & -120627 & 0.020 & 11.43 & 10.34 & -1.0 & 45.7 & 0.54 & \\
\hline NGC 0232 & 004246.5 & -233331 & 0.020 & 11.51 & 10.71 & 1.1 & 55.7 & 0.66 & $2006 e t$ \\
\hline NGC 3110 & 100402.7 & -062835 & 0.017 & 11.29 & 10.94 & 3.3 & 33.0 & 0.39 & \\
\hline NGC 5010 & 131225.4 & -154745 & 0.021 & 10.84 & 9.79 & -1.0 & 11.8 & 0.14 & \\
\hline NGC 5331 & 135216.6 & +020608 & 0.033 & 11.60 & 10.92 & 3.0 & 67.1 & 0.79 & \\
\hline NGC 6240 & 165258.6 & +022403 & 0.024 & 11.81 & 10.89 & -0.3 & 108.4 & 1.28 & $2000 b g, \mathbf{2 0 1 0 g p}$ \\
\hline NGC 6926 & 203304.8 & -020139 & 0.020 & 11.25 & 11.38 & 5.6 & 30.4 & 0.36 & \\
\hline NGC 7130 & 214819.6 & -345705 & 0.016 & 11.34 & 10.75 & 1.2 & 37.1 & 0.44 & 2010bt \\
\hline NGC 7592 & 231822.2 & -042456 & 0.024 & 11.36 & 10.51 & -1.0 & 38.4 & 0.45 & \\
\hline NGC 7674 & 232756.9 & +084646 & 0.029 & 11.37 & 10.92 & 1.1 & 40.0 & 0.47 & 2000A, 2011ee, $2011 \mathrm{hb}$ \\
\hline
\end{tabular}

Notes. The last column reports our $6 \mathrm{SNe}$ (in bold) with other SNe discovered previously in the galaxy sample.

last column we list (in boldface) the designation of the SNe discovered in our search that are the basis for our analysis. For completeness we also list (in italics) the SNe discovered by other SN searches outside our monitoring period. The distribution of $L_{B}$ and $L_{\mathrm{TIR}}$ are compared in Fig. 1 showing that, as is typical of $\mathrm{SBs}, L_{\mathrm{TIR}}$ is on average a factor ten higher than $L_{B}$, whereas $L_{\mathrm{TIR}} \sim L_{B}$ for normal star-forming galaxies. We notice that almost all galaxies are LIRGs, and only two are ULIRGS. Most galaxies of the sample are isolated $(\sim 60-70 \%)$ while the remaining are double/interacting galaxies, or they contain double nuclei, a signature of a recent merger. Several galaxies of the sample are asymmetrical or disturbed, or they show warps, bars, and tidal tails.

\subsection{Search strategy}

To search SNe in the selected SB sample we used the HAWK-I instrument installed at the ESO VLT telescope at Cerro Paranal (Chile). HAWK-I is an NIR $(0.85-2.5 \mu \mathrm{m})$ wide-field imager with a mosaic of four Hawaii-2RG detectors. The total field of view is $7.5^{\prime} \times 7.5^{\prime}$ with a scale of $0.106^{\prime \prime} /$ pix. Even in poor seeing conditions $(>1.5 \operatorname{arcsec})$, the instrument allows $S / N \sim 10$ for a $K=20$ mag star with a 15 min exposure.

The infrared light curves of SNe evolve relatively slowly, remaining within one or two magnitudes from maximum for two or three months (Mattila \& Meikle 2001) and therefore an IR SN search does not require frequent monitoring. We planned for an average of three visits per galaxy per semester, for a total of 80

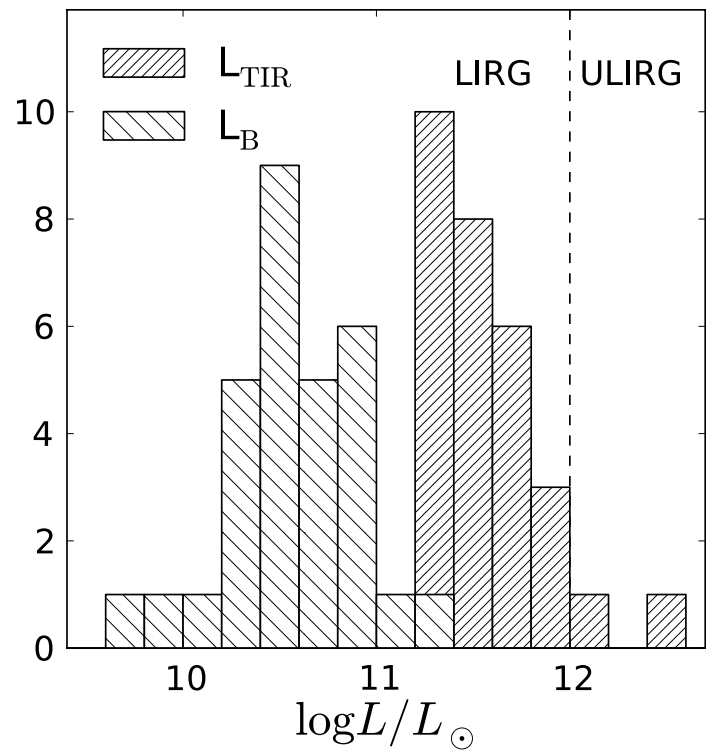

Fig. 1. Distribution of the B and FIR luminosities for the SB galaxies of our sample.

to 100 visits. The monitoring campaign was scheduled in service mode and we did not set tight constraints for the sky conditions. This and the relatively short duration of the observing blocks made the program well suited as filler. We notice that we had no 
influence on the actual scheduling of the observations that had to follow the rules of the ESO service-mode scheduler.

Eventually, the fraction of useful observing time was $100 \%$ of the allocated time in the first season, and $70 \%$ in the second and third semesters. The log of the observations is reported in Table 2 where for each galaxy we list the epoch of observations (MJD), the seeing (FWHM in arcsec), and the minimum and maximum magnitude limits for SN detection across the image (cf. Sect. 2.5). In total, we obtained $210 \mathrm{~K}$-band exposures (exposure time $15 \mathrm{~min}$ ), with an average of about three visits per galaxy per semester. Because of the time loss, three galaxies were not monitored in the last two seasons.

It turned out that the average image quality was quite good: for $\sim 90 \%$ of the exposures, the seeing was less than $1.0^{\prime \prime}$, with an average FWHM across the whole program of $0.6^{\prime \prime}$.

\subsection{Data reduction and analysis}

For data reduction and mining of the HAWK-I mosaic images, we developed a custom pipeline that integrates different, publicly available recipes and tools in a Python environment.

The pipeline consists of four sections:

1. prereduction, astrometric calibration, and production of the stacked mosaic image. For these steps we used the ESO HAWK-I pipeline recipes in EsoRex, the ESO Recipe Execution Tool'

2. subtraction of images taken at different epochs using ISIS (Alard 2000) for the PSF matching;

3. search for transient candidates in the difference image using Sextractor (Bertin \& Arnouts 1996). The candidates were ranked based on their Sextractor-measured parameters and submitted to the operator for visual inspection and validation;

4. estimate of the detection efficiency through artificial star experiments performed for each of the search images (details in Sect. 2.5).

The raw images were retrieved from the ESO archive as soon as they became available, and immediately reduced to allow for activation of follow-up spectroscopy of transient candidates.

For the prereduction, we followed the reduction cascade described in the HAWK-I pipeline manual ${ }^{3}$ including dark subtraction, flat-field and illumination corrections, background subtraction, distortion correction, astrometric offset refinement, combination of the different exposures, and stitch of the four detectors in a single mosaic image. Actually, it turned out that the ESO pipeline recipes for background subtraction and offset refinement do not provide satisfactory results for our images. The main reason is the extended size of our sources and the consequent large dithering we had adopted. To address this issue we implemented custom recipes for the two aforementioned reduction steps.

The most critical step in the data reduction is image subtraction, in particular in the proximity of the nuclear regions of the galaxies. First of all we need to choose a proper reference image, usually the image with the best seeing obtained at least three month before (or in some case after) the image to be searched. We also need to choose the proper parameters for the image difference procedure (see Melinder et al. 2012 for an extensive discussion). An additional problem arises because in the distributed

\footnotetext{
2 http://www.eso.org/sci/software/cpl/esorex.html

$3 \mathrm{ftp}: / /$ ftp.eso.org/pub/dfs/pipelines/hawki/

hawki-pipeline-manual-1.8.pdf
}
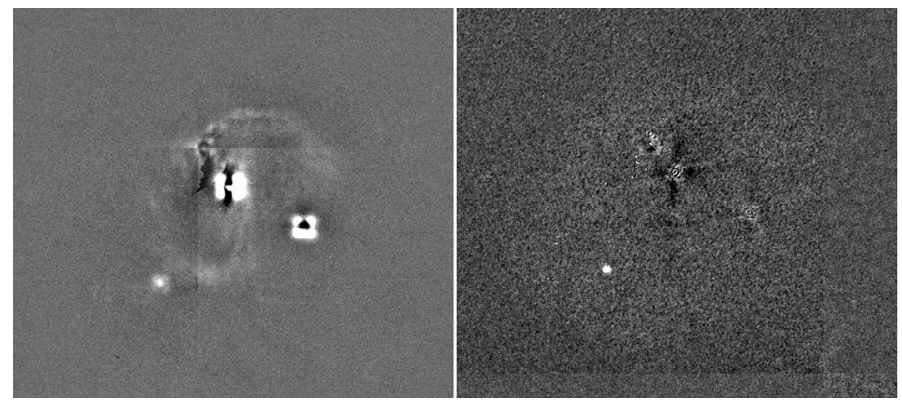

Fig. 2. Left panel: example of poor subtraction of images of NGC 7130 with large seeing differences $\left(F W H M=1.5^{\prime \prime}\right.$, with $F W H M=0.4^{\prime \prime \prime}$ for the reference). Right panel: optimal subtraction for two images with similar good seeing $\left(F W H M=0.4^{\prime \prime}\right)$. In both panels the source in the lower left quadrant is SN 2010bt (cf. Fig. 3). The FOV in both panels is about $2^{\prime} \times 2^{\prime}$.

version of ISIS, the program automatically selects the reference sources for computating the convolution kernel. Owing to the small number of sources in our extragalactic fields, the reference source list in general includes the bright galaxy nucleus, which, being very bright, has a significant weight in the determination of the kernel. This may cause some problems, because if at one epoch an SN occurs very close to the galaxy nucleus, it can be included in the convolution kernel and effectively cancelled in the difference image. We therefore modified the ISIS selection procedure to allow for excluding specific sources, in particular the galaxy nuclei, from the reference list.

Despite efforts in many cases the difference image shows significant spurious residuals that correspond to the galaxy nuclear regions. The problem is most severe in case of images with poor seeing $\left(F W H M>1^{\prime \prime}\right)$ and/or reduced transparency. This is illustrated in Fig. 2 where we show two examples of image difference, one for a search image with poor seeing $(F W H M=$ $1.5^{\prime \prime}$, left panel) and the other for a case with excellent seeing $\left(F W H M=0.4^{\prime \prime}\right)$. In both cases, the reference image was the same and had excellent seeing $\left(F W H M=0.4^{\prime \prime}\right)$.

False detections due to residuals of the image subtraction were largely removed by the requirement that the candidate had to be visible at least in two consecutive epochs.

\subsection{Supernova discoveries and characterization}

During our monitoring campaign six transients were detected in at least two consecutive epochs separated by at least one month (finding charts are in Fig. 3). Four of them were spectroscopically confirmed as SNe (three SN-CC and one SN Ia), and we argue in the following that the other two transients, labeled as probable SN (PSN), are also likely SN CC (Table 3). SNe 2010bt and 2010gp were discovered and announced before our detection by optical searches but have been independently rediscovered by us.

The objects are listed in Table 3 along with the host galaxy name, distance modulus (computed from the galactocentric redshift and the adopted cosmology), SN coordinates, offsets from the galaxy nucleus, and projected linear distances from the galaxy nucleus.

For all transients, $K$-band magnitudes were measured through aperture photometry on the difference images and calibrated with respect to 2MASS stars in the field. Upper limits measured on prediscovery images were also estimated. For all transients, but PSN2010 in IC 4687, we obtained some follow-up imaging in the optical or near-infrared domains. These 
Table 2. Log of the observations.

\begin{tabular}{|c|c|c|c|c|c|c|c|c|c|c|c|c|c|c|c|}
\hline \multicolumn{4}{|c|}{ CGCG011-076 } & \multicolumn{4}{|c|}{ ESO440-IG058 } & \multicolumn{4}{|c|}{ IRAS17207-0014 } & \multicolumn{4}{|c|}{ NGC 0232} \\
\hline JD & Seeing & $\mathrm{m}_{\max }^{\lim _{2}}$ & $\lim _{\min }$ & JD & Seeing & $\mathrm{m}_{\max }^{\lim }$ & $\lim _{\min }$ & JD & Seeing & $\mathrm{m}_{\max }^{\lim }$ & $\lim _{\min }$ & 2 & S. & $\mathrm{m}_{\max }^{\lim _{2}}$ & $\mathrm{~m}_{\min }^{\lim }$ \\
\hline 55189.3 & 0.41 & 19.0 & 13.5 & 989.0 & 0.36 & 19.0 & 15.5 & 035.0 & 39 & .0 & 16.5 & 15.3 & 34 & 19.0 & 6.0 \\
\hline & & & & & 0.43 & 19.0 & & 056.0 & 37 & 9.0 & & & & & .0 \\
\hline & 0.65 & 19.0 & 3.1 & 339.0 & 0.56 & 19.0 & 17.3 & 090.9 & .56 & 9.0 & 15.3 & & . 05 & 19.0 & 4.2 \\
\hline \multicolumn{4}{|c|}{ CGCG043-099 } & 55701.9 & 0.49 & 19.0 & 18.8 & 55342.3 & 0.78 & 19.0 & 14.2 & & NGC & 3110 & \\
\hline $\mathrm{JD}$ & Seeing & $\mathrm{m}_{\max }^{\lim }$ & $\lim _{\min }$ & 55734.1 & 1.8 & 18.3 & 15.5 & 55398.1 & 1.16 & 18.7 & 16.7 & $\mathrm{JD}$ & Seeing & $\mathrm{m}_{\max }^{\lim }$ & $\mathrm{m}_{\min }^{\lim }$ \\
\hline 54989.1 & 0.49 & 19.0 & 13.5 & \multicolumn{4}{|c|}{ ES0507-G070 } & 55415.2 & 0.49 & 19.0 & 14.8 & 55184.3 & 0.47 & 19.0 & 16.0 \\
\hline 55019.1 & 0.92 & 19.0 & 13.0 & JD & Seeing & $\mathrm{m}_{\max }^{\lim }$ & $\mathrm{m}_{\min }^{\lim }$ & 55446.1 & 1.05 & 19.0 & 16.0 & 55202.3 & 0.35 & 19.0 & 16.8 \\
\hline 55238.3 & 0.98 & 19.0 & 13.1 & 4989.1 & 0.36 & 19.0 & 16.5 & 55675.3 & 0.42 & 9.0 & 18.0 & 55291.0 & 0.45 & 19.0 & 5.8 \\
\hline & 116 & 190 & 12.5 & 55016.1 & 053 & 100 & 16.7 & 55739.3 & & 8.5 & & & 0.56 & & 6.7 \\
\hline & 0.42 & 19.0 & 135 & .2 & 0.36 & 19.0 & 16.5 & 55775.0 & 66 & 9.0 & 17.0 & & 0.68 & .0 & 5.0 \\
\hline & SO148-1 & G002 & & 55251.3 & 0.53 & 19.0 & 16.5 & 55796.1 & 1.10 & 18.7 & 17.3 & 55708.0 & 0.42 & 19.0 & 5.5 \\
\hline JD & Seeing & $\mathrm{m}_{\max }^{\lim }$ & $\mathrm{m}_{\min }^{\lim }$ & 55284.0 & 0.47 & 19.0 & 16.7 & \multicolumn{4}{|c|}{ IRAS18090+0130 } & 55189.3 & 0.33 & 19.0 & 16.2 \\
\hline 54990.3 & 0.68 & 19.0 & 15.0 & & IC 162 & & & JD & Seeing & $\mathrm{m}_{\max }^{\lim }$ & $\lim _{\min }$ & & NGC & 5010 & \\
\hline & 0.69 & 19.0 & 15.0 & JD & Seeing & $\mathrm{m}_{\max }^{\lim }$ & $\mathrm{m}_{\min }^{\lim }$ & 55019.1 & 1.19 & 19.0 & 16.0 & JD & Seeing & $\mathrm{m}_{\max }^{\lim }$ & $\mathrm{m}_{\min }^{\lim }$ \\
\hline & 0.42 & 19.0 & 15.0 & 5056.3 & 0.41 & $\frac{\max }{19.0}$ & $\frac{15}{15.5}$ & 55038.1 & 051 & 19.0 & 16.7 & 55014.9 & 0.50 & $\frac{\operatorname{mix}}{19.0}$ & $\frac{\min }{16.0}$ \\
\hline & 0.5 & 18.7 & 15.0 & .3 & & 19 & 15.5 & & & & 16.2 & & & & 4.5 \\
\hline & & & & & & & & & & & & & & & \\
\hline & 1. & 1 & 1 & 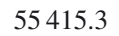 & & 19 & 16.6 & & & & & & & & .7 \\
\hline & & & 0 & 3 & & & & & & & & & & & 4.5 \\
\hline & & & & & & & & & & & & & & & \\
\hline & & 1 & 15.0 & 3 & 0. & 19 & 15.3 & 55 & 0 & & 1 & & & & .5 \\
\hline 3.1 & 0.59 & 18.8 & 16.8 & .1 & 0.63 & 18.8 & 14.8 & 55672.3 & 0.39 & 19.0 & 15.7 & & NGC & 5331 & \\
\hline & SO239 & 3002 & & & IC 25. & & & 55742.2 & 0.47 & 19.0 & 15.3 & 然 & Seeing & $\mathrm{m}_{\max }^{\lim _{2}}$ & . \\
\hline JD & Seeing & $\mathrm{m}_{\max }^{\lim }$ & $\mathrm{m}_{\min }^{\lim }$ & . & Seeing & $\mathrm{m}_{\max }^{\lim }$ & $\lim _{\min }$ & & 0.89 & 18.5 & $15=2$ & 5016.1 & 049 & 19.0 & 14.0 \\
\hline 55019.2 & 0.72 & $\frac{1 \ln x}{19.0}$ & $\frac{100}{14.7}$ & 5137.3 & 0.9 & 19 & 15.5 & & CG-02-1 & $1-051$ & & 3 & 5 & 7 & .8 \\
\hline 55028.2 & 0.57 & 19.0 & 14.5 & 55172.3 & 0.4 & 19.0 & 15.5 & JD & Seeing & $\mathrm{m}_{\max }^{\lim }$ & $\mathrm{m}_{\mathrm{min}}^{\lim }$ & 55263.3 & 0.48 & 19.0 & 15.0 \\
\hline & & & 14.5 & & & & 16.0 & 55037.4 & & 19.0 & $\frac{\min }{18.5}$ & & & & 5.3 \\
\hline & & 15 & 14.5 & & 0.5 & 12 & 155 & & & & 18.8 & & 0.42 & 17.1 & 65 \\
\hline 55365.2 & 0.69 & 19.0 & 15.5 & & IC 28 & & & 55068.4 & 0.47 & 19.0 & 18.2 & & NGC & 6240 & \\
\hline 98.1 & 1.16 & 18.7 & 14.5 & JD & Seeing & $\mathrm{m}_{\max }^{\lim }$ & $\mathrm{m}_{\mathrm{min}}^{\lim }$ & 55398.2 & 0.92 & 19.0 & 18.8 & JD & Seeing & $\mathrm{m}_{\max }^{\lim }$ & $\mathrm{m}_{\mathrm{min}}^{\lim }$ \\
\hline & & & 14.7 & 502 & & & 14.0 & & & & & & & & $\frac{m}{4.7}$ \\
\hline & & & & 5522 & & & 13.8 & & & & & & & & \\
\hline & & & 14.5 & 2 & 0 & 19 & 14.8 & & & & & & & & 5.8 \\
\hline & 1.58 & 18.2 & 14.8 & & & & & & & 19.0 & 1 & & 0.8 & & 3 \\
\hline & $\overline{\mathrm{ESO} 244}$ & $\overline{\mathrm{CO1}} \mathrm{Y}$ & & JI & Seeing & $\mathrm{m}_{\max }^{\lim }$ & $m$ & & $\overline{03-1}$ & 44-014 & & 0 & 1.00 & 17.7 & 2.3 \\
\hline JD & Seeing & $m_{\max }^{\lim }$ & $\mathrm{m}_{\min }^{\lim }$ & 54989.1 & 0.37 & $\frac{\ln 2 \mathrm{x}}{19.0}$ & $\begin{array}{l}\min \\
16.0\end{array}$ & S & eing & $\mathrm{m}_{\max }^{\lim }$ & $\mathrm{m}_{\mathrm{min}}^{\lim }$ & & 0.36 & 18.2 & 1.0 \\
\hline 55028.3 & & $\frac{10}{190}$ & $\frac{130}{13.0}$ & 550560 & & 19.0 & 15.8 & 056.3 & 18 & 19.0 & $\frac{\min }{17.0}$ & & & & 3.0 \\
\hline & & & & & & & & & & & & & & & \\
\hline & & & & & & & & & & & & & & & 4.7 \\
\hline & & & & & & 19 & 16.2 & & & & & & 1. & 19.0 & 3.5 \\
\hline & 1 & & 14.0 & 553 & 1 & 19.0 & 15.3 & & 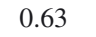 & 9.0 & 14.8 & & $\mathbf{N}$ & 6926 & \\
\hline & 0.8 & 18.8 & 13.5 & 3.3 & 0.66 & 19.0 & 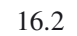 & 2.1 & & v & 15.0 & & Seeing & $\mathrm{m}_{\max }^{\lim }$ & $\mathrm{m}_{\mathrm{min}}^{\lim }$ \\
\hline 55705.3 & 1.26 & 18.5 & 15.5 & & $\overline{A S 1222}$ & $4-0624$ & & 55402.2 & 1.16 & 19.0 & 14.5 & 55068.1 & 0.44 & 19.0 & 14.8 \\
\hline 55757.3 & 0.84 & 19.0 & 16.2 & JD & Seeing & $\mathrm{m}_{\max }^{\lim }$ & $\mathrm{m}_{\mathrm{min}}^{\lim }$ & 55415.4 & 0.39 & 19.0 & 14.8 & 55366.3 & 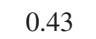 & 19.0 & 15.0 \\
\hline & ESO26 & 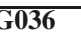 & & 8000 & & 19.0 & 16.5 & & 0 & $\overline{34}$ & & & 1.2 & 18.5 & 4.0 \\
\hline JD & 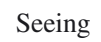 & $\mathrm{m}^{\lim }$ & $\mathrm{m}_{\min }^{\lim }$ & & & 19 & 16.0 & & & $\mathrm{~m}_{\max }^{\lim }$ & & & 0.3 & 0 & 63 \\
\hline 142.3 & 0.00 & 18.0 & 14.0 & 55226.2 & 0.47 & 19.0 & 16.0 & 55049.3 & & 18.5 & 14.3 & & 0. & 19.0 & 6.7 \\
\hline 2.3 & 0.6 & 18.3 & 14.5 & & AS1437 & $8-3651$ & & 55068.3 & 0.5 & 18.8 & 14.0 & & 0. & -5 & 5.5 \\
\hline & 0.4 & 18.7 & 14.0 & JD & Seeing & $\mathrm{m}_{\max }^{\lim }$ & $\mathrm{m}_{\min }^{\lim }$ & & 1.3 & .2 & 12 & & 0.45 & 19.0 & 7.7 \\
\hline 9.0 & 0.5 & 18.3 & 13.5 & 54990.1 & 0.53 & $\frac{\operatorname{limax}}{19.0}$ & $\frac{\mathrm{min}}{13.5}$ & 55449.3 & 0.4 & 19.0 & 13.5 & & & 7130 & \\
\hline & 0.55 & 19.0 & 16.8 & 55072.9 & 0.36 & 19.0 & 14.5 & 55704.4 & 0.79 & 19.0 & 14.0 & & Seeing & $\mathrm{m}_{\max }^{\lim }$ & $\mathrm{m}_{\mathrm{min}}^{\lim }$ \\
\hline 55701.9 & 0.50 & 19.0 & 17.5 & & AS1639 & $9-0937$ & & 55739.2 & 1.00 & 19.0 & 15.3 & 55038.1 & 0.47 & 19.0 & 16.0 \\
\hline 55734.0 & 1.80 & 18.8 & 16.7 & JI & Seei & $\mathrm{m}_{\max }^{\lim }$ & in $2>$ & 55771.3 & 0.59 & 18.8 & 14.2 & & 0.76 & 19.0 & 14.7 \\
\hline & SO286 & & & 5014 & 0.4 & 19 & 16.0 & & & & & & 0 & 19.0 & 6.3 \\
\hline JI & & & & & 0.4 & 19. & 16.7 & JD & ig & $\mathrm{m}_{\max }^{\lim }$ & & & 0 & 19.0 & 16.7 \\
\hline 54988.4 & $0 \Omega$ & & 15.0 & 55057.0 & 0. & 19.0 & 16.3 & 55068.1 & & 19.0 & 14.5 & & 0.40 & 5 & 13.7 \\
\hline & & & & & & & & & & & & & & & 5.3 \\
\hline & & & & & & & 15 & & & & 13 & & 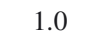 & 19.0 & 6.0 \\
\hline 55056.1 & 0.4 & 19.0 & 15.5 & 55398.0 & 1.20 & 18.7 & 15.7 & 55446.1 & 0.84 & 19.0 & 14.0 & & NGC & 7592 & \\
\hline & & 10 & 14.5 & 55416.1 & 0.4 & 19.0 & 16.5 & & 0.68 & 19.0 & 15.7 & & Seeing & $\mathrm{m}_{\max }^{\lim }$ & $\mathrm{m}_{\min }^{\lim }$ \\
\hline & & & & & & 19 & 16.3 & & 0.17 & 19.0 & & 5398.2 & & & 3.8 \\
\hline & 0.6 & & & & 0.6 & 19.0 & 17 & & NGC & 232 & & & 0.37 & & 4.2 \\
\hline & 0.8 & & 15.0 & 55710.0 & 0.6 & 18.7 & 16.3 & JD & Seeing & $\mathrm{m}_{\max }^{\lim }$ & $\mathrm{m}_{\mathrm{min}}^{\lim }$ & 55444.2 & 0.40 & 19.0 & 15.0 \\
\hline & & & & & & 18 & 15 & 55090.1 & & 19.0 & 15.2 & & & & 3.5 \\
\hline & & & & & & & 16 & & & & & & & & 5.5 \\
\hline 735.2 & 1.60 & 19.0 & 14.5 & 55795.0 & 0.87 & 18.9 & 16.2 & 55402.2 & 0.95 & 19.0 & 16.0 & 55796.3 & 0.65 & 19.0 & 13.5 \\
\hline
\end{tabular}

Notes. The table includes the epoch of observations (MJD), the seeing (FWHM in arcsec), and the minimum and maximum magnitude limits for SN detection across the image (cf. Sect. 2.5). 


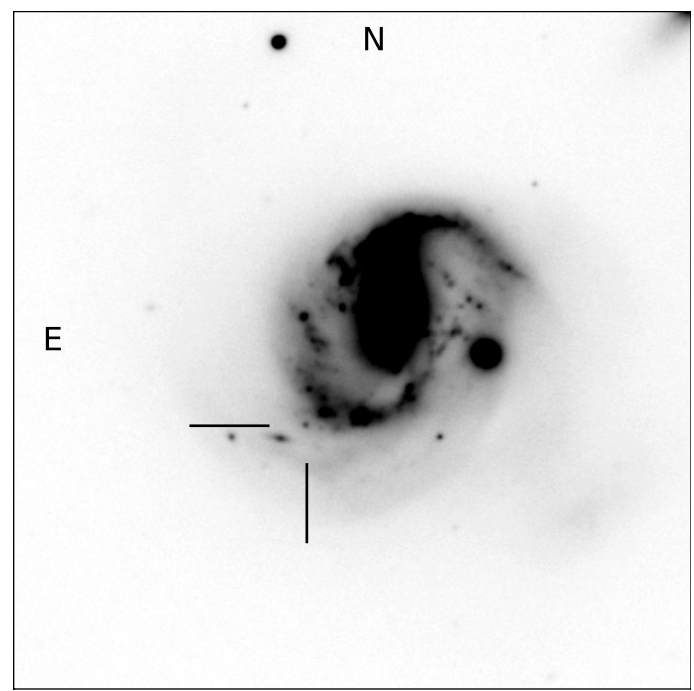

(a) SN 2010bt

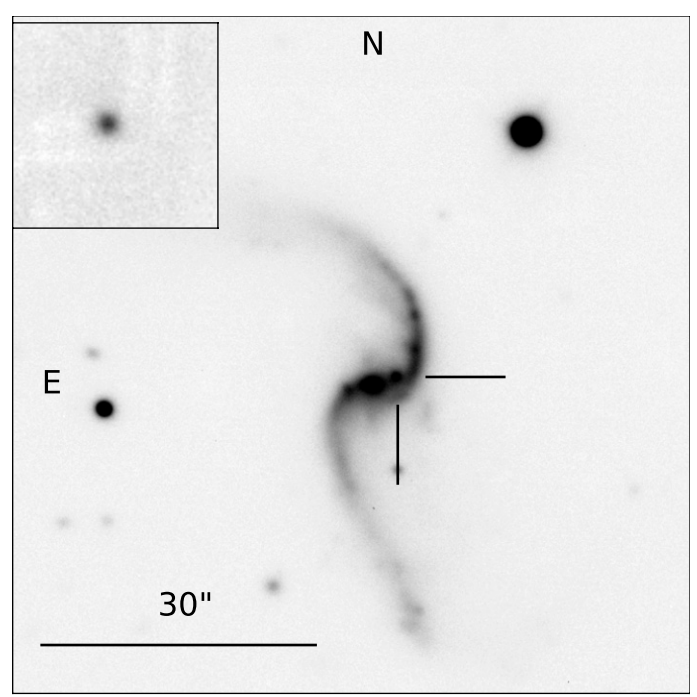

(c) SN 2010hp

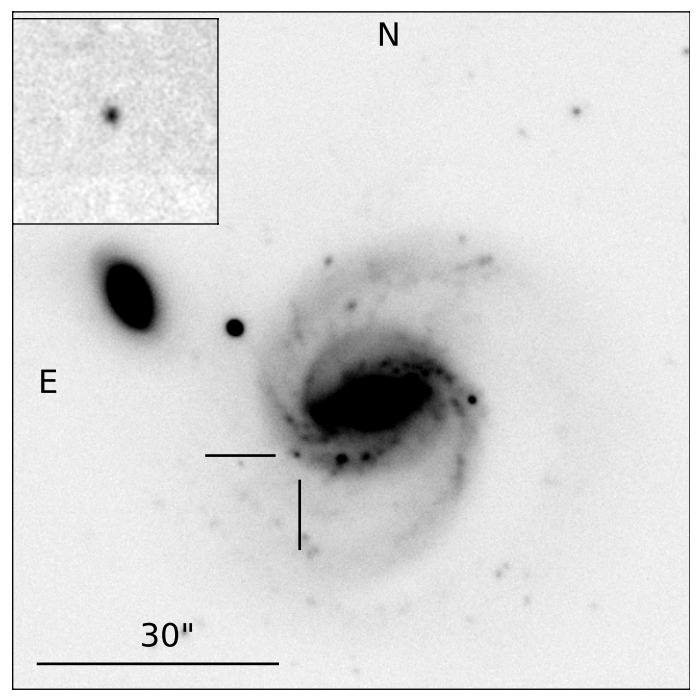

(e) SN 2011ee

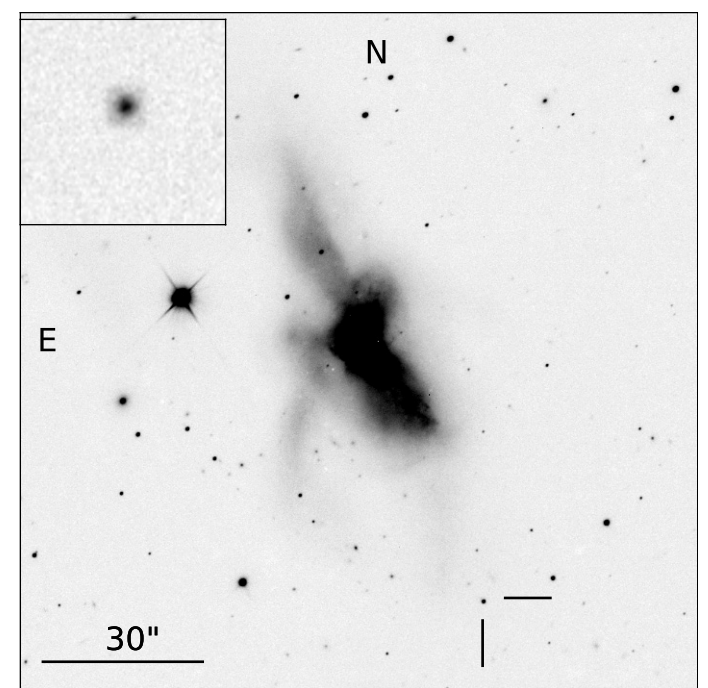

(b) SN 2010gp

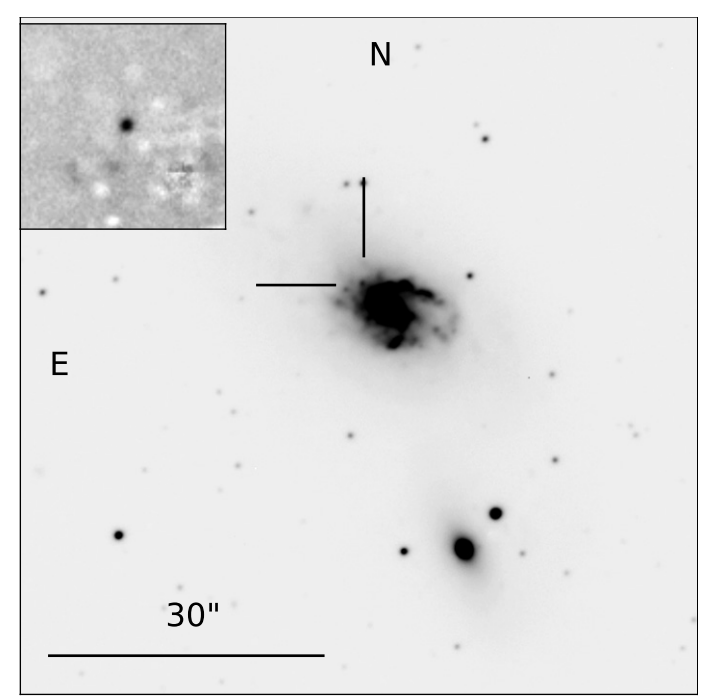

(d) PSN2010 in IC 4687

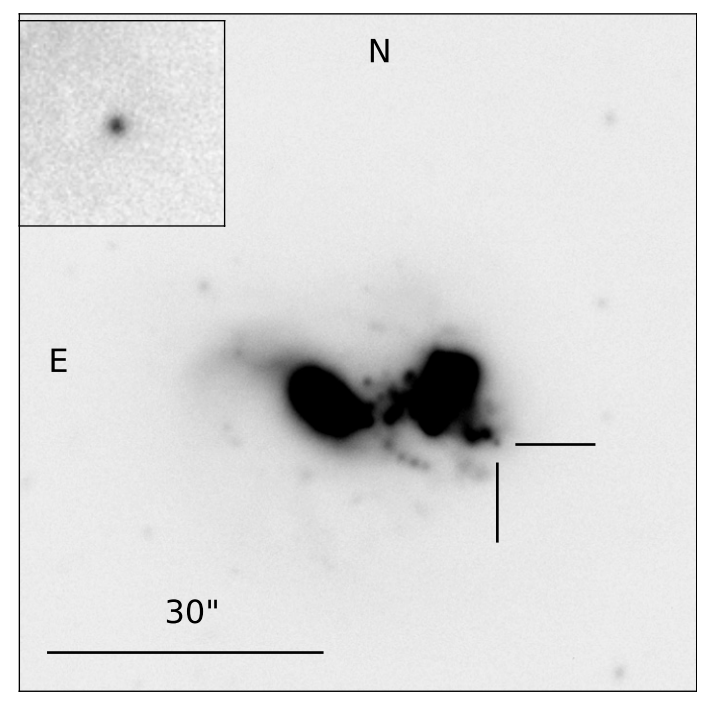

(f) PSN 2011 in IC 1623A

Fig. 3. $K$-band finding charts for the $\mathrm{SNe}$ of our list. The inserts show the transients as they appear in the difference image. 
Table 3. Information for the detected SNe.

\begin{tabular}{|c|c|c|c|c|c|c|}
\hline & SN2010bt & SN2010gp & SN2010hp & SN2011ee & PSN2010 & PSN2011 \\
\hline Host galaxy & NGC 7130 & NGC 6240 & MCG-02-01-52 & NGC 7674 & IC 4687 & IC $1623 \mathrm{~A}$ \\
\hline Distance modulus & 34.08 & 35.07 & 35.24 & 35.39 & 34.21 & 34.54 \\
\hline $\mathrm{RA}(\mathrm{SN})$ & $21: 48: 20.22$ & $16: 52: 57.39$ & $0: 18: 50.01$ & $23: 27: 57.34$ & $18: 13: 40.213$ & $01: 07: 46.229$ \\
\hline Dec. $(\mathrm{SN})$ & $34: 57: 16.5$ & $2: 23: 16.4$ & $-10: 21: 40.6$ & $+08: 46: 38.10$ & $-57: 43: 28.00$ & $-17: 30: 29.48$ \\
\hline Offset ["] & $9 \mathrm{E} 14 \mathrm{~S}$ & $22 \mathrm{~W} 47 \mathrm{~S}$ & $2.6 \mathrm{~W} 2.7 \mathrm{~N}$ & $9.3 \mathrm{E} 6.3 \mathrm{~S}$ & $2.6 \mathrm{E} 2.8 \mathrm{~N}$ & $4 \mathrm{E} 7 \mathrm{~S}$ \\
\hline$r[\mathrm{Kpc}]$ & 5.3 & 25.9 & 2.1 & 8.9 & 6.5 & 3.2 \\
\hline SN type & IIn & Ia & IIP & Ic & IIP" & $\mathrm{Ic}^{\#}$ \\
\hline$R \max [\mathrm{mag}]$ & $15.9 \pm 0.1$ & $16.0 \pm 0.1$ & $17.6 \pm 0.2$ & $17.3 \pm 0.5$ & & $16.9 \pm 0.5$ \\
\hline$K \max [\mathrm{mag}]$ & $16.1 \pm 0.2$ & $16.9 \pm 0.1$ & $17.2 \pm 0.1$ & $18.0 \pm 0.5$ & $17.9 \pm 0.2$ & $17.0 \pm 0.3$ \\
\hline$A B_{\mathrm{G}}[\mathrm{mag}]$ & 0.44 & 0.33 & 0.16 & 0.25 & 0.43 & 0.07 \\
\hline$A B_{\mathrm{H}}[\mathrm{mag}]$ & $1.7 \pm 0.5$ & $0.2 \pm 0.1$ & $0.5 \pm 0.3$ & $\sim 0.0$ & $0-8$ & $0.5 \pm 0.5$ \\
\hline abs $M_{R}$ & -19.2 & -19.2 & -17.9 & -18.1 & & -17.9 \\
\hline $\operatorname{abs} M_{K}$ & -18.1 & -18.2 & -18.0 & -17.7 & $-16.3 /-17.3$ & -17.5 \\
\hline MJD max R & $55303 \pm 5$ & $55405 \pm 3$ & $55399 \pm 5$ & $55760 \pm 5$ & $55270 \pm 20$ & $55725 \pm 10$ \\
\hline Refs. & CBET 2250,2252 & CBET 2388, 2390 & CBET 2246, 2249 & CBET 2773 & & \\
\hline
\end{tabular}

Notes. \# Photometric classification.

observations were reduced using standard procedures in IRAF. When a reference image was not available, the SN magnitude was measured using the PSF fitting technique. Optical band magnitudes were calibrated with respect to Landolt's standard fields. Our photometry for the six transients is reported in Table 4. For the two transients with no spectroscopic confirmation, the photometry is used to assess their nature.

Spectroscopic observations were obtained for four candidates: epoch, spectral range, and instruments are reported in Table 5. Data were reduced using standard procedure in IRAF but for the $\mathrm{X}$-shooter spectra that were reduced using version 1.0.0 of the ESO X-shooter pipeline (Goldoni et al. 2006) with the calibration frames (biases, darks, arc lamps, and flat fields) taken during daytime. The extracted spectra, after wavelength and flux calibration, were compared with a library of template spectra using the GELATO SN spectra comparison tool ${ }^{4}$ (Harutyunyan et al. 2008). The best fit template SN, the SN type, and phase are reported in Table 5. Spectroscopic classification for PSN2010 in IC 4687 was attempted, but the observed spectrum turned out to be too noisy for a safe classification. The table includes the result of the spectroscopic observations of SN 2010gp from Folatelli et al. (2010).

We have used the available photometry and spectroscopy to put some constraints on the amount of extinction suffered by the $\mathrm{SNe}$. Hereafter, we describe the sparse information available for each transient in some details.

SN 2010bt was discovered on 2010 April 17.10 UT by Monard (2010). A spectrum taken on April 18.39 UT (Turatto et al. 2010) shows strong resemblance to several type-IIn SNe, in particular SN 1996L (Benetti et al. 1999) shortly after explosion (Fig. 4). A broad $\mathrm{H}_{\alpha}$ component is present, indicating an expansion velocity of about $3500 \mathrm{~km} \mathrm{~s}^{-1}$ (half width at zero intensity). SN 2010bt was independently rediscovered by us on May 25 and was observed at other two epochs. The object was not visible on a HAWK-I image taken in 2009 July 26 (limit $K=19.0 \mathrm{mag}$ ). While the analysis of the light curve and spectral evolution of this SN will be presented elsewhere Elias-Rosa et al. (in prep.), a preliminary analysis shows that to match the color of SN 2010bt to that of the type IIn SN 1998S requires a significant amount of

\footnotetext{
4 https://gelato.tng.iac.es/
}

extinction $\left(A_{B}=1.7 \pm 0.5 \mathrm{mag}\right)$. This extinction improves also the matching of the spectra of SN 2010bt to SN 1996L. SN 2010gp was discovered on 2010 July 14.10 UT by Maza et al. (2010) with the 0.41-m PROMPT1 telescope located at Cerro Tololo. Folatelli et al. (2010) reported the spectroscopic classification as a type-Ia SN around maximum light with high expansion velocity of the ejecta. SN 2010gp was rediscovered independently by us on July 21 and was observed at other two epochs. The object was not visible on a HAWK-I image taken on 2010 May 26 (limit $K=18.5 \mathrm{mag}$ ). The available colors compared to that of standard SN Ia suggest a small reddening inside the host galaxy, $A_{B}=0.2$ mag.

SN 2010hp was discovered on a HAWK-I image taken on 2010 July 21.3 UT (Miluzio \& Cappellaro 2010). The object was not detected on 2009 Aug. 25 (limit $K=19.0$ mag). Based on a spectrum taken on 2010 Sept. 8, the SN was classified as a type II event more than 30 days past maximum light (Marion et al. 2010). We obtained a follow-up spectrum on 2010 Sept. 15 with EFOSC2 at the NTT showing the typical features of type II SN: that is, H Balmer lines, NaI D doublet, and the NIR CaII emission triplet along with a number of FeII lines in the blue region. The GELATO spectral comparison tool found a best match with the type IIP SN 1999em (Elmhamdi et al. 2003) at about +60 days, adopting a reddening of about $A_{B}=0.5 \mathrm{mag}$ (Fig. 4). This is fully consistent with the color curve comparison between the two SNe.

SN 2011ee was discovered on 2011 June 27.3 UT (Miluzio et al. 2011). The object was not detected on a $K$-band image taken on 2010 September 7 ( $K>19.0 \mathrm{mag})$. An optical spectrum was obtained with X-shooter at the VLT on 2011 July 17.3 UT showing that the transient is a type Ic SN. The GELATO code finds a best match with SN 2007gr (Hunter et al. 2009) at maximum (Fig. 4). Because the classification of type Ic SNe near maximum is sometimes ambiguous we obtained a follow-up spectrum about two months later (2011 Sept. 20) using OSIRIS at the GTC. Again the spectrum is very similar to SN $2007 \mathrm{gr}$ at a corresponding phase (Fig. 4 bottom panel). The color comparison and the spectral match are consistent with a negligible host galaxy extinction.

PSN2010 in IC 4687 was discovered on 2010 May 21.3 UT in the northern component of a galaxy triplet that include IC 4686 and, 1 arcmin to the south of IC 4687, IC 4689. IC 4687 has a chaotic structure formed by stars, gas, dust, 
Table 4. Transient photometry.

\begin{tabular}{|c|c|c|c|c|c|c|c|c|}
\hline MJD & $B$ & $V$ & $R$ & $I$ & $J$ & $H$ & $K$ & Instr. \\
\hline \multicolumn{9}{|c|}{ SN 2010bt } \\
\hline 55341.4 & & & & & & & $16.9(.1)$ & $\mathrm{H}$ \\
\hline 55366.4 & & & & & & & $18.08(.1)$ & $\mathrm{H}$ \\
\hline 55382.3 & & & & & & & $18.68(.1)$ & $\mathrm{H}$ \\
\hline \multicolumn{9}{|c|}{ SN 2010gp } \\
\hline 55342.4 & & & & & & & $>18.5$ & $\mathrm{H}$ \\
\hline 55398.1 & & & & & & & $17.4(.1)$ & $\mathrm{H}$ \\
\hline 55416.1 & & & & & & & $16.85(.1)$ & $\mathrm{H}$ \\
\hline 55457.0 & & & & & & & $17.9(.1)$ & $\mathrm{H}$ \\
\hline 55455.0 & $19.72(.05)$ & $18.43(.03)$ & $18.11(.03)$ & $18.15(.03)$ & & & & $\mathrm{E}$ \\
\hline \multicolumn{9}{|c|}{ 2010hp } \\
\hline 55398.3 & & & & & & & $17.25(.05)$ & $\mathrm{H}$ \\
\hline 55415.4 & & & & & & & $17.17(.05)$ & $\mathrm{H}$ \\
\hline 55449.4 & & & & & & & $17.20(.10)$ & $\mathrm{H}$ \\
\hline 55454.3 & $19.61(.03)$ & $18.70(.03)$ & $18.28(.03)$ & $17.89(.03)$ & & & & $\mathrm{E}$ \\
\hline 55456.1 & & & & & $17.24(.1)$ & $16.93(.1)$ & $17.17(.1)$ & $\mathrm{S}$ \\
\hline 55498.1 & & & & & $18.48(.1)$ & $18.51(.1)$ & $17.99(.1)$ & $\mathrm{S}$ \\
\hline 55499.2 & $>19.1$ & $19.61(.03)$ & $18.83(.03)$ & $18.83(.03)$ & & & & $\mathrm{E}$ \\
\hline 55563.1 & $>20.8$ & $>20.0$ & $>18.5$ & $19.50(.05)$ & & & & $\mathrm{E}$ \\
\hline 55737.3 & & & $>20.4$ & $>20.1$ & & & & $\mathrm{E}$ \\
\hline \multicolumn{9}{|c|}{ 2011ee } \\
\hline 55739.3 & & & & & & & $18.6(.2)$ & $\mathrm{H}$ \\
\hline 55752.3 & & & $18.50(.1)$ & & & & & $\mathrm{R}$ \\
\hline 55771.4 & & & & & & & $18.4(.1)$ & $\mathrm{H}$ \\
\hline 55775.4 & & & & & & & $18.5(.1)$ & $\mathrm{H}$ \\
\hline 55796.3 & & & & & & & $19.2(.1)$ & $\mathrm{H}$ \\
\hline 55808.3 & $>19.72(0.05)$ & $18.43(0.03)$ & $18.11(0.03)$ & $18.15(0.03)$ & & & & $\mathrm{E}$ \\
\hline \multicolumn{9}{|c|}{ PSN 2010 } \\
\hline 55056.1 & & & & & & & $>19.3$ & $\mathrm{H}$ \\
\hline 55336.2 & & & & & & & $17.98(.10)$ & $\mathrm{H}$ \\
\hline 55337.2 & & & & & & & $17.99(.10)$ & $\mathrm{H}$ \\
\hline 55338.2 & & & & & & & $17.91(.10)$ & $\mathrm{H}$ \\
\hline 55366.3 & & & & & & & $17.85(.10)$ & $\mathrm{H}$ \\
\hline 55375.1 & & & & & & & $18.42(.3)$ & $\mathrm{H}$ \\
\hline 55393.3 & & & & & & & $18.77(.3)$ & $\mathrm{H}$ \\
\hline \multicolumn{9}{|c|}{ PSN2011 } \\
\hline 55762.4 & & & & & & & $18.15(.2)$ & $\mathrm{H}$ \\
\hline 55774.7 & & $>19.30$ & $19.50(.2)$ & $19.0(.2)$ & & & & $\mathrm{D}$ \\
\hline 55796.4 & & & & & & & $18.72(.2)$ & $\mathrm{H}$ \\
\hline 55808.4 & $>21.30$ & $>20.0$ & $20.10(.3)$ & $19.50(.3)$ & & & & $\mathrm{E}$ \\
\hline 55812.2 & & & & & & & $19.43(.2)$ & $\mathrm{H}$ \\
\hline
\end{tabular}

Notes. Estimated errors are given in parentheses. H=HAWK-I@VLT, E=EFOSC2@NTT, S=SOFI@NTT, L=RATCam@Liverpool, D= Dolores@TNG

Table 5. Log of spectroscopic observations.

\begin{tabular}{lccccccc}
\hline \hline SN & MJD & Range $(\mathrm{nm})$ & Res. $(\mathrm{nm})$ & Instrument & Best fit & Type & Phase \\
\hline 2010bt & 55304.4 & $350-1000$ & 1.4 & EFOSC/NTT & $2005 \mathrm{gj}$ & IIn & $\max$ \\
2010hp & 55454.2 & $350-1000$ & 1.4 & EFOSC/NTT & 1999em & Ia & $+60 \mathrm{~d}$ \\
2010 IC 4687 & 55352.2 & $340-1000$ & 0.2 & XSHOOTER/VLT & \multicolumn{2}{c}{ S/N too low } \\
2011ee & 55824.6 & & 2.5 & OSIRIS/GTC & 2007gr & Ic & +60 \\
2011ee & 55759.3 & $340-1000$ & 0.2 & XSHOOTER/VLT & 1994I & Ic & $\max$ \\
2010gp & 55401.0 & $354-886$ & Folatelli et al. (2010) & 2002bo & Ia & just before max \\
\hline
\end{tabular}

and a large curly tail. The transient was not detected on a $K$-band image taken on 2009 Aug. 8.1 ( $K>19.0 \mathrm{mag})$. We obtained an optical/infrared spectrum with X-shooter at VLT on 2010 June 5. However, because of its very low S/N, we could not derive a convincing classification, therefore we had to rely on the $K$-band photometry. Comparing the $K$ band absolute light curve of PSN2010 $\left(A_{B}(\right.$ host $\left.)=0\right)$ with template light curves of different SN types we found a good match with SN 2005cs, a prototype of underluminous type IIP SN (Pastorello et al. 2009), assuming that the detection of PSN2010 was two months after the explosion. However, lacking color measurements, we could not constrain the extinction, and indeed assuming a high extinction $A_{B} \sim 8 \mathrm{mag}$, we found an alternative good match with the 


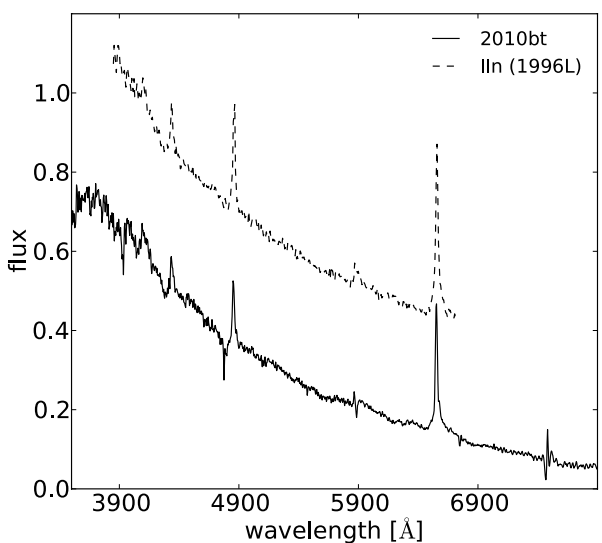

(a)

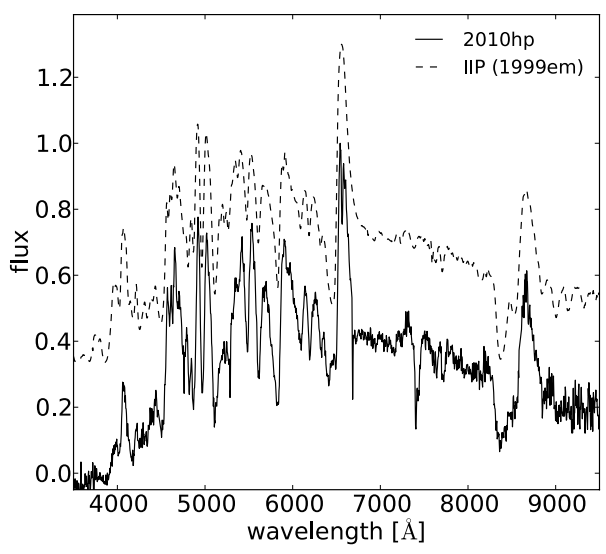

(b)

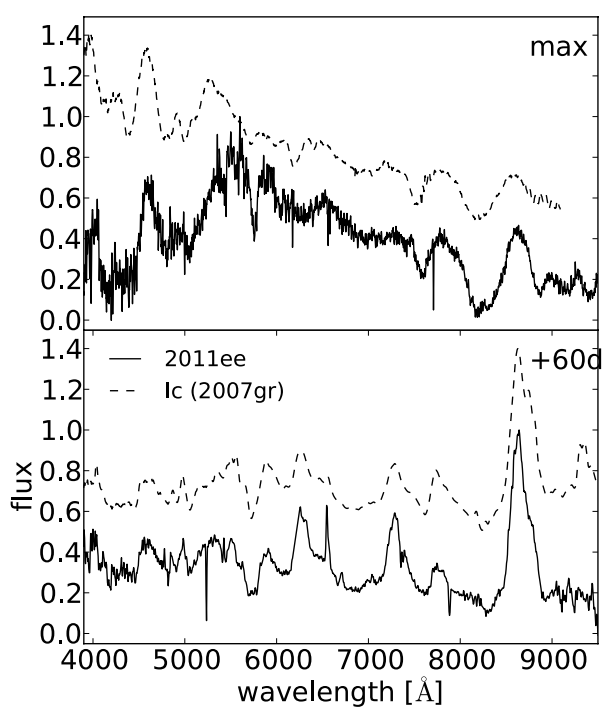

(c)

Fig. 4. Spectra of SN 2010bt, 2010hp, and 2011ee are shown along with the best-fitting templates. a) The spectrum of 2010bt, dereddened by $A_{B}=1.7 \mathrm{mag}$ (see text) is compared with that of the type IIn SN 1996L. b) The spectrum of $2010 \mathrm{hp}$, dereddened by $A_{B}=0,5 \mathrm{mag}$ (see text) is compared with that of the type IIP SN $1999 \mathrm{em}$. c) The spectrum of 2011ee is compared to that of the SN Ic $2007 \mathrm{gr}$ at the maximum (top panel) and 60 days after the maximum.

light curve of SN 1999em (Fig. 5). Intermediate values may also be adopted by fitting other SN II.

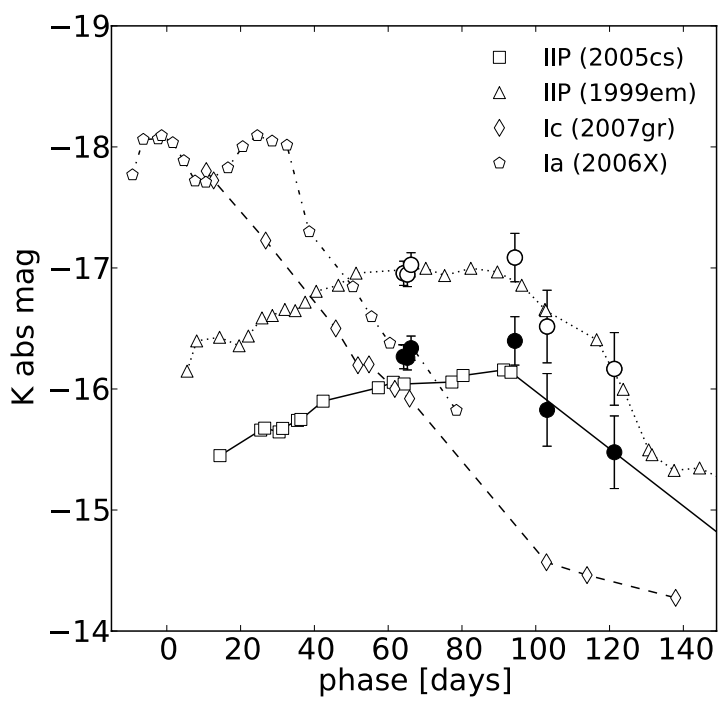

Fig. 5. $K$ band absolute light curve of the PSN2010 (black dots), compared with those of template SNe. The empty circles show the same data but assume an extinction $A_{B}=8 \mathrm{mag}$.

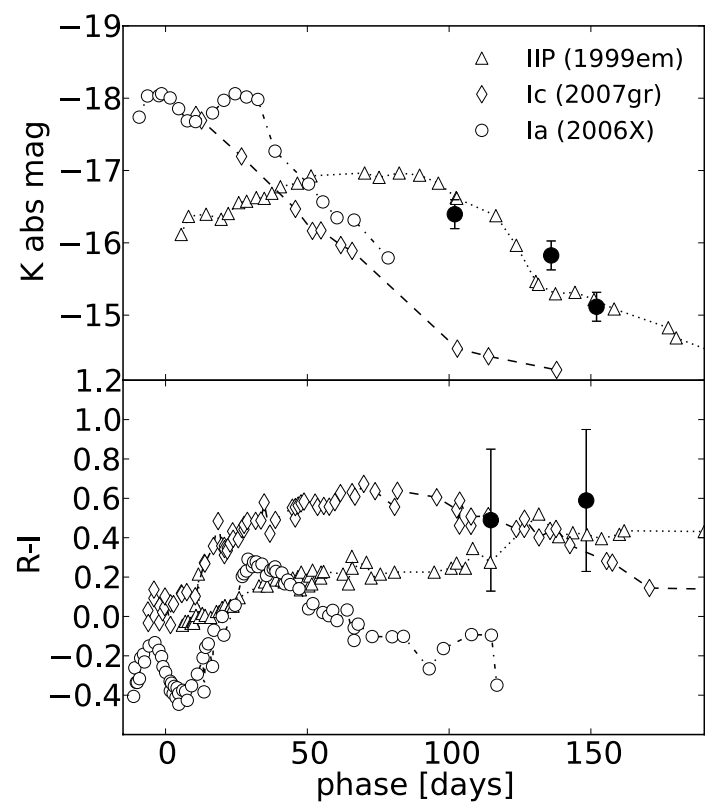

Fig. 6. $K$ and $R-I$ colors of PSN 2011, compared with those of template SNe. For this plot we adopted $A_{B}=0.5 \mathrm{mag}$, which improves the fit of the $R$ band photometry ( $\Delta R=0.3 \mathrm{mag})$.

PSN2011 in IC 1623 was discovered on 2011 July 21.4 UT in the western component of a galaxy pair. The object was not detected on a $K$-band image taken on 2010 Sept. 5 ( $K>19.0 \mathrm{mag}$ ). Unfortunately, due to bad weather in the scheduled nights, we could not obtain a spectroscopic observation of the transients. We had to rely on three epochs of photometry, in K complemented by two epochs in the optical $R$ and $I$ bands. A simultaneous comparison of the absolute observed luminosity with template $\mathrm{SNe}$ gives a best fit with the SN Ic 2007gr one month after maximum (Fig. 6). Assuming this classification, from the colors we can constrain the extinction to be $A_{B}=0.5 \pm 0.5 \mathrm{mag}$. However, we have to admit that, within the errors, the photometry of PSN2011 also can be consistent with a type IIP at about three months after explosion. 
To summarize, during the search we discovered six SNe. Four received spectroscopic classifications: one as a type Ia and three as core collapse events, a type IIn, a type IIP, and a type Ic. For the other two, based on the sparse photometry, we argue that they are most likely core collapse SNe, with a best fit as types IIP and Ic, respectively.

\subsection{Search detection limit}

To derive the SN rate from the number of detected events it is crucial to obtain an accurate estimate of the magnitude detection limit for each of the search images and for different locations in the images. As shown in Fig. 2, the detection efficiency is influenced by the sky conditions at the time of observations (namely seeing and transparency) and by the transient position inside the host galaxy.

The magnitude limit for SN detection has been estimated through artificial star experiments. The procedure we adopted was the following:

1. fake SNe of different magnitudes are simulated with the PSF derived from isolated field stars;

2. the image is segmented into a number of intensity contour levels. We took denser contours in the nuclear regions because the magnitude limit changes rapidly with background intensity;

3. one fake $\mathrm{SN}$ of specific magnitude is randomly placed inside a chosen intensity contour;

4. the image with the fake $\mathrm{SN}$ is processed through the image difference and transient detection pipeline;

5. if the fake $\mathrm{SN}$ results in a detected transient, the experiment is repeated with a fainter artificial star until we have a null detection. The fainter magnitude for which the fake SN is detected defines the magnitude limit for the given background intensity level;

6. Steps 2 to 5 were repeated for each contour level three times to enhance the statistical significance of the results. The average value for each contour level has been adopted as the magnitude discovery limit for the given background intensity.

To illustrate the results, a plot of the magnitude limit versus background counts for four observations of the galaxy NGC 7130 is shown in Fig. 7. Each epoch is labeled with the image seeing, while the errorbar shows the range of limiting magnitudes for the three experiments. The top $x$-axis shows the linear distance in $\mathrm{Kpc}$ from the galaxy center.

It can be seen that, as expected, the magnitude limit is lower in the nuclear regions, which for a typical galaxy, correspond to $1.5-2.0 \mathrm{kpc}$. Epochs with different seeing have similar magnitude limits in the galaxy outskirts (typically $K \sim 19 \mathrm{mag}$ ), while in the nuclear region when seeing is poorer the magnitude limit is brighter (in the worst case even 5-6 mag brighter than in the galaxy outskirts).

\section{SN search simulation}

To evaluate the significance of the detected events we elaborated a simulation tool that returns the number and properties of expected events based on specific features of our SN search, including a number of parameters describing our current knowledge of SBs and SN properties. The tool uses a MonteCarlo approach that simulates the stochastic nature of SN explosions. By collecting several MonteCarlo experiments with the same input parameters, we can test whether the observed events are within

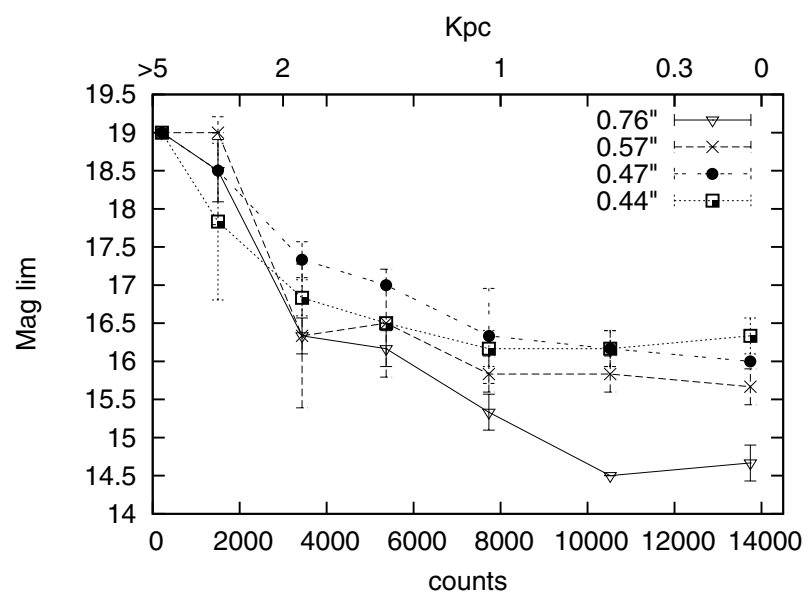

Fig. 7. Magnitude limit vs. background intensity for four different observations of NGC 7130. The points are labeled with the respective image seeing. Errorbars show the range of the magnitude limits from the three different experiments.

the expected distribution. On the other hand, by varying some of the input parameters, we can test the influence of specific assumptions.

\subsection{The simulation tool}

Our MonteCarlo (MC) simulation tool was built in a Python environment and makes use of standard values taken from the literature for the different inputs. For those that are more controversial, we give references with some discussion. The basic ingredients of the simulation are:

- relevant data for the selected SBs, namely: redshift, galactic extinction, infrared fluxes from IRAS catalogs at 25,60 , and $100 \mu, B$ magnitude corrected for internal extinction ${ }^{5}$, Hubble morphological type. These data were retrieved from NED;

- information describing the SN properties for each of the SN types considered here: SNe Ia and core collapse events, including SNe IIP, IIL, IIn, and Ib/c. $K$-band template light curves were constructed starting from $B$ template light curves (Cappellaro et al. 1997) and $B-K k$-corrections for the given galaxy redshift (Botticella et al. 2008). We note that the results of this procedure agree closely with the $K$ template light curves of Mattila \& Meikle (2001). For the SN luminosity functions we adopted those of Li et al. (2011b) as reference, but we also tested for the possible presence of a significant population of faint core collapse, which may be suggested by the analysis of very nearby SNe (Horiuchi et al. 2011). From the LOSS project we also adopted the relative rates for the different SN types (Li et al. 2011a);

- details of the search campaign: log of observations, magnitude detection limit for each observation as a function of the host galaxy background intensity;

- the number of SNe expected from a given star formation episode. For core collapse SNe, this is determined only by the adopted mass range of the progenitors and IMF slope. In fact, for our purposes, we can neglect the very short time delay from $\mathrm{CC}$ progenitor formation to explosion. For type Ia $\mathrm{SNe}$, we need to consider the realization factor, which is the fraction of events in the proper mass range, which occurs in

5 The galaxy internal extinction was retrieved from HyperLeda (Paturel et al. 2003). 
suitable close binary systems and the delay-time distribution (Sect. 3.1.2);

- the depth and distribution of the extinction by dust inside the parent galaxies (cf. Sect. 3.1.3);

- the star formation spatial distribution in the parent galaxies (cf. Sect. 3.1.4).

Hereafter, we discuss our assumptions about the parameters of the simulation.

\subsubsection{From IRAS measurements to star formation rate}

The SFR in SBs can be estimated on the basis of the galaxy total infrared luminosity $\left(L_{\mathrm{TIR}}\right)$ under the assumption that dust reradiates a major fraction of the UV luminosity and after calibration with stellar synthesis models. The TIR luminosity can, in turn, be estimated from FIR flux measurements.

Helou et al. (1988) provided a prescription for deriving the FIR emission from IRAS measurements:

$\mathrm{FIR}=1.26 \times 10^{-14}\left[2.58 f_{v}(60 \mu \mathrm{m})+f_{v}(100 \mu \mathrm{m})\right]$

where FIR is in $\mathrm{W} \mathrm{m} \mathrm{m}^{-2}$ and $f_{v}$ are in Jansky. FIR fluxes are converted into TIR fluxes by using the relation of Dale et al. (2001)

$\log \frac{\mathrm{TIR}}{\mathrm{FIR}}=a_{0}+a_{1} x+a_{2} x^{2}+a_{3} x^{3}+a_{4} x^{4}$

where $x=\log \frac{f_{v}(60 \mu \mathrm{m})}{f_{v}(100 \mu \mathrm{m})}$ and $[a(z=0)] \simeq[0.2378,-0.0282$, $0.7281,0.6208,0.9118]$.

The TIR fluxes are converted in luminosities using the adopted distances:

$L_{\mathrm{TIR}}=4 \pi D^{2} \mathrm{TIR}$

Finally, the relation between the SFR $(\psi)$ and $L_{\mathrm{TIR}}$ was derived by Kennicutt (1998) from SB galaxy spectral synthesis model adopting 10-100 Myr continuous bursts and a Salpeter IMF as

$\frac{\psi}{\left[M_{\odot} \mathrm{yr}^{-1}\right]}=\frac{L_{\mathrm{TIR}}}{2.2 \times 10^{43}\left[\mathrm{erg} \mathrm{s}^{-1}\right]}=\frac{L_{\mathrm{TIR}}}{5.8 \times 10^{9}\left[L_{\odot}\right]}$.

\subsubsection{SNR and SFR}

In general, the rate of $\mathrm{SNe}$ expected at a specific time, $\dot{n}_{\mathrm{SN}}(t)$, for a stellar population depends on the star formation history, the number of $\mathrm{SNe}$ per unit mass from one stellar generation (labeled as SN productivity), and the distribution of delay time from star formation to explosion for the specific SN type. Following the notation of Greggio $(2005,2010)$,

$\dot{n}_{\mathrm{SN}}(t)=\int_{0}^{t} \psi(t-\tau) k_{\mathrm{SN}} f_{\mathrm{SN}}(\tau) \mathrm{d} \tau$

where $\psi(t)$ is the SFR, $f_{\mathrm{SN}}$ is the distribution of the delay times $\tau$ and $k_{\mathrm{SN}}$ is the $\mathrm{SN}$ productivity. The equation shows that at a fixed epoch $t$ since the beginning of star formation, the rate of SNe is obtained by adding the contribution of all past stellar generations, each of them weighted with the SFR at the appropriate time.

\section{Core collapse SNe}

For core collapse SNe the delay time from star formation to explosion (2.5 Myr for $120 M_{\odot}$ stars up to $40 \mathrm{Myr}$ for $8 M_{\odot}$ stars) is shorter than the typical SB duration (200-400 Myr, McQuinn et al. 2009). Assuming that the SFR in the SB was constant during the past $40 \mathrm{Myr}$, the expected $\mathrm{CC} \mathrm{SN}$ rate, $\dot{n}_{\mathrm{CC}}$, is proportional to the current SFR:

$\dot{n}_{\mathrm{CC}}=k_{\mathrm{CC}} \times \psi$.

The SN productivity $k_{\mathrm{CC}}$ is derived by integrating the IMF, $\phi(m)$, and assuming a $\mathrm{CC}$ progenitor mass $\left(M_{\mathrm{CC}}\right)$ range:

$k_{\mathrm{CC}}=\frac{\int_{M_{\mathrm{CC}}^{\mathrm{L}}}^{M_{\mathrm{U}}^{\mathrm{U}}} \phi(m) \mathrm{d} m}{\int_{M_{\mathrm{L}}}^{M_{\mathrm{U}}} m \phi(m) \mathrm{d} m}$.

where $M_{\mathrm{CC}}^{\mathrm{L}}$ and $M_{\mathrm{CC}}^{\mathrm{U}}$ are respectively the lower and upper mass limits for SN CC progenitors, and $M_{\mathrm{L}}$ and $M_{\mathrm{U}}$ are the lower and upper stellar mass limit. To be consistent with the Kennicutt SFR calibration we adopted a Salpeter IMF, that is,

$\phi(m) \propto m^{-\alpha} \quad$ with $\alpha=2.35$ and $0.1 M_{\odot}<M<100 M_{\odot}$.

Assuming $8<M_{\mathrm{CC}}<50 M_{\odot}$ for the $\mathrm{CC}$ progenitor mass range, $k_{\mathrm{CC}}=0.007 \mathrm{M}^{-1}$. This number changes significantly if we adopt a different IMF, e.g. $k_{\mathrm{CC}}=0.011$ for a Kroupa IMF or $k_{\mathrm{CC}}=0.039$ for an extreme Starburst IMF (Dwek et al. 2011). We soon note, however that, because the IMF also enters into the conversion from $L_{\mathrm{TIR}}$ to $\psi$, the expected rate of $\mathrm{SN}$ events is almost independent of the selected IMF (cf. Sect. 4.1), provided the choice is consistent.

More important is the assumption on the mass range for $\mathrm{CC}$ progenitors, which is not well constrained. Actually, while changing the upper limit of the progenitor mass from 40 to $100 M_{\odot}$ makes a modest $10 \%$ increase in the CC SN productivity, the lower mass limit is crucial, with $k_{\mathrm{CC}}$ decreasing by $30 \%$ if we adopted $M_{\mathrm{CC}}^{\mathrm{L}}=10 M_{\odot}$ instead of the favored value of $8 M_{\odot}$ (Smartt 2009).

\section{SN la}

Estimating the expected rate of SN Ia is complicated because the delay time distribution $f_{\text {Ia }}$, while still uncertain, certainly ranges from short to a very long time. In particular, it has been suggested that SN Ia can be divided into two classes, one with a short delay time whose rate scales with the current SFR (also called prompt), and a second with a long delay time (tardy), whose rate scales with the average of the SFR during the entire galactic evolution (Scannapieco \& Bildsten 2005; Mannucci et al. 2006). While stellar evolution arguments (Greggio 2010, 2005; Greggio \& Renzini 1983) and more recent data (Maoz et al. 2012; Totani et al. 2008) suggest a continuous distribution of the delay time instead of two distinct classes, the schematization is still a fair approximation that helps in simplifying the problem of predicting the expected SN Ia rate in SBs.

In general, for a galaxy of the local Universe, $\sim 13 \mathrm{Gyr}$ after the beginning of SFR, we can identify the contribution of the two components as follows (Greggio 2010):

$\dot{n}_{\mathrm{Ia}}(13)=k_{\mathrm{Ia}} \times\left(\psi_{C} \int_{0}^{0.1} f_{\mathrm{Ia}}(\tau) \mathrm{d} \tau+\psi_{P} \int_{0.1}^{13} f_{\mathrm{Ia}}(\tau) \mathrm{d} \tau\right)$

where $\psi_{C}$ and $\psi_{P}$ are the average SFR over, respectively, the last 0.1 Gyr (current SFR) and from 0.1 to 13 Gyr ago (past SFR). 
The SN productivity $k_{\mathrm{Ia}}$ is the product of the number of stars per unit mass in the adopted progenitor mass range $(0.021$ for a Salpeter IMF and a mass range $3 M_{\odot}<M<8 M_{\odot}$ ) and the realization fraction, the actual fraction of systems which make a successful explosion ( $\sim 5 \%$ according to the most recent estimate) (Maoz \& Mannucci 2012). We assume that SF history in SBs can be described schematically with two components: a constant SFR during the galaxy evolution that created the galaxy stellar mass, and an ongoing episode of intense SFR that is the source of the strong TIR emission. Neglecting the contribution of the ongoing SB to the galaxy stellar mass, we can approximate $\psi_{P} \simeq M / 13 \times 10^{9}$ and write Eq. (2) as

$\dot{n}_{\mathrm{Ia}}(13) \simeq k_{\mathrm{Ia}}\left(\psi_{C} F_{\mathrm{Ia}}^{p}+\frac{M}{13 \times 10^{9}} F_{\mathrm{Ia}}^{t}\right)$

where $F_{\mathrm{Ia}}^{p}=\left\langle f_{\mathrm{Ia}}^{p}\right\rangle \times 0.1$ and $F_{\mathrm{Ia}}^{t}=\left\langle f_{\mathrm{Ia}}^{t}\right\rangle \times 13 \simeq 1-F_{\mathrm{Ia}}^{p}$ are the relative fraction of prompt and tardy events derived by integrating the delay time distribution in the relevant time range. In our approximation, $\psi_{C}$ can be derived from the observed $L_{\mathrm{TIR}}$ and the galaxy mass from the $K$ magnitude and $B-K$ colors (cf. Mannucci et al. 2005).

The relative contribution of the two SN Ia components has been a very debated issue in the past few years, ranging from $F_{\text {Ia }}^{p} \sim 50 \%$ (Mannucci et al. 2006) to $F_{\text {Ia }}^{p} \sim 10 \%$ from standard stellar evolution scenarios (Greggio 2010). In our simulation we adopted an intermediate value, $F_{\mathrm{Ia}}^{p} \sim 30 \%$ as reference.

\subsubsection{Extinction}

Dust extinction in SBs is very high, especially in the nuclear regions. For instance, Shioya et al. (2001) find that fitting the spectral energy distribution of the nuclear region of Arp 220 requires a visual extinction $A_{V}>30 \mathrm{mag}$. Actually, according to Engel et al. (2011), "over most of the disk the near-infrared obscuration is moderate, but increases dramatically in the central tens of parsecs of each nucleus". Similar high extinction, $A_{V} \sim 20$, was found for the SB region of Zw 096 (Inami et al. 2010).

As a first-order approximation, for our simulation we assumed that the extinction has the same distribution of the SF (see next section) with a maximum value $A_{V}=30$ mag corresponding to the SFR peak and scaled linearly in the other regions. While this is a crude approximation, it turns out that the actual choice of extinction correction has little impact on our simulation. In the nuclear, high-extinction regions, the SN detection is limited by the reduced performance of the image subtraction algorithm in these high surface-brightness regions. At the same time, our IR search is largely insensitive to variation in the (moderate) extinction of the outer galaxy regions.

For the wavelength dependence of extinction, we adopted the Calzetti's law with $R_{V}=4.05 \pm 0.8$ (Calzetti et al. 2000).

\subsubsection{Star formation distribution}

The spatial distribution of the SFR is a key ingredient of the simulation. This is because we expect that SNe occur more frequently in the high SF regions where, on the other hand, our detection efficiency is lower. In principle, the FIR emission that is used to estimate the SFR would also be a good tracer of its spatial distribution. However, it turned out that the available MIR imaging for the galaxies of our sample (mainly obtained with the Spitzer observatory) does not have enough spatial resolution for mapping the compact SB structures.

Selected $K$-band images from our survey can have excellent resolution, but as is well-known, the near IR emission traces the
Table 6. Morphological classification, FIR colors, and compactness classification for the SBs of our sample.

\begin{tabular}{lcccc}
\hline \hline Galaxy & $\begin{array}{c}\text { Morp. } \\
\text { class }\end{array}$ & $\log f_{60} / f_{100}$ & $\log f_{25} / f_{60}$ & $\begin{array}{c}\text { Comp. } \\
\text { class }\end{array}$ \\
\hline CGCG011-076 & $\mathrm{S}$ & -0.20 & -0.89 & 3 \\
CGCG043-099 & $\mathrm{S}$ & -0.19 & -1.05 & 3 \\
ESO148-IG002 & $\mathrm{CP}$ & 0.01 & -0.82 & 2 \\
ESO239-IG002 & $\mathrm{M}$ & -0.04 & -0.79 & 2 \\
ESO244-G012 & $\mathrm{CP}$ & -0.10 & -0.68 & 2 \\
ESO264-G036 & $\mathrm{S}$ & -0.34 & -0.96 & 4 \\
ESO286-IG019 & $\mathrm{M}$ & 0.07 & -0.81 & 2 \\
ESO440-IG058 & $\mathrm{P}$ & -0.23 & -0.99 & 3 \\
ESO507-G070 & $\mathrm{S}$ & -0.08 & -1.21 & 1 \\
IC 1623A/B & $\mathrm{CP}$ & -0.14 & -0.80 & 2 \\
IC 2545 & $\mathrm{M}$ & 0.01 & -0.88 & 2 \\
IC 2810 & $\mathrm{P}$ & -0.22 & -1.00 & 3 \\
IC 4687/6 & $\mathrm{P}$ & -0.13 & -0.76 & 2 \\
IRAS12224-0624 & $\mathrm{S}$ & -0.14 & -1.48 & 1 \\
IRAS14378-3651 & $\mathrm{S}$ & -0.08 & -1.00 & 1 \\
IRAS16399-0937 & $\mathrm{CP}$ & -0.24 & -0.87 & 3 \\
IRAS17207-0014 & $\mathrm{M}$ & -0.05 & -1.36 & 1 \\
IRAS18090+0130 & $\mathrm{P}$ & -0.31 & -0.98 & 4 \\
MCG-02-01-051/2 & $\mathrm{P}$ & -0.14 & -0.79 & 2 \\
MCG-03-04-014 & $\mathrm{S}$ & -0.15 & -0.91 & 3 \\
NGC 0034 & $\mathrm{M}$ & 0.01 & -0.85 & 2 \\
NGC 0232 & $\mathrm{P}$ & -0.23 & -0.89 & 3 \\
NGC 3110 & $\mathrm{P}$ & -0.30 & -1.00 & 4 \\
NGC 5010 & $\mathrm{S}$ & -0.33 & -0.85 & 4 \\
NGC 5331 & $\mathrm{CP}$ & -0.29 & -1.00 & 4 \\
NGC 6240 & $\mathrm{CP}$ & -0.06 & -0.81 & 2 \\
NGC 6926 & $\mathrm{S}$ & -0.31 & -0.83 & 4 \\
NGC 7130 & $\mathrm{P}$ & -0.19 & -0.89 & 3 \\
NGC 7592 & $\mathrm{CP}$ & -0.12 & -0.92 & 2 \\
NGC 7674 & $\mathrm{P}$ & -0.19 & -0.45 & 2 \\
\hline
\end{tabular}

Notes. Following Hattori et al. (2004), type 1 have SF region $<500$ pc, type $2<1 \mathrm{Kpc}$, type $3>1 \mathrm{Kpc}$ and type 4 have extranuclear star formation.

old star population better, which is the galaxy mass distribution more than the SFR distribution. Therefore for an estimate of the SFR concentration, we are forced to an indirect, statistical approach.

Our starting point is the SB classification by Hattori et al. (2004), who derived a correlation between the global SBs properties, such as FIR colors, and the compactness of the SF regions. These range from very compact $(\leq 100 \mathrm{pc})$ nuclear starbursts with almost no star-forming activity in the outer regions (type 1), to extended starbursts with relatively faint nuclei (type 4), with types 2 and 3 as intermediate cases. In addition, they find a trend for galaxies with more compact SF region to show a higher star formation efficiency and hotter far-infrared color. They also find that the compactness of SF regions is weakly correlated with the galaxy morphology, with disturbed objects primarly showing more concentrated SF. On the other hand, an appreciable fraction $(\sim 50 \%)$ of their galaxy sample was dominated by extended starbursts (type 4). The significant variations in the degree of concentration of the SB SF regions has been recently confirmed by McQuinn et al. (2012).

For the reference simulation we used the input parameters summarized in Table 7. In the next section we compare the prediction of the simulation with the current SN discoveries. In an attempt to characterize the SF spatial distribution for the SBs of our sample, we derived estimates of their morphological class and FIR colors. In particular, following Hattori et al. (2004), 


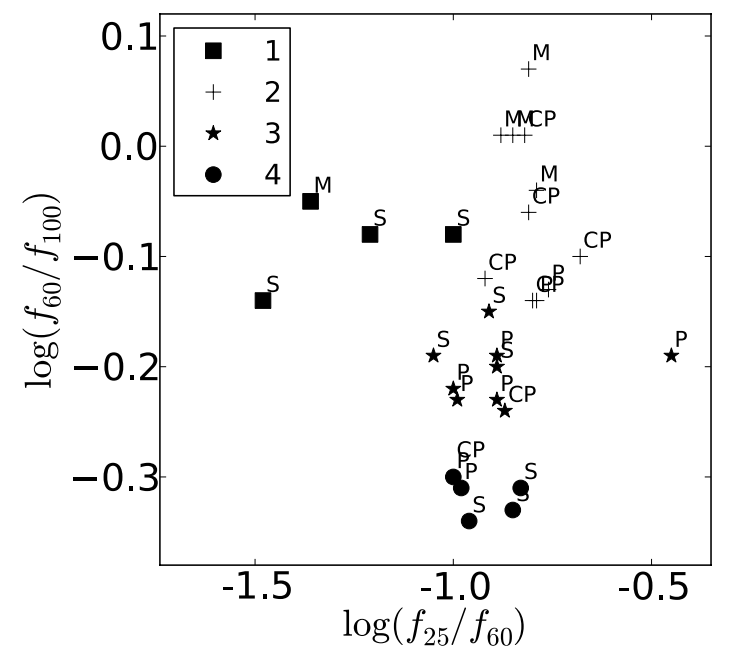

Fig. 8. FIR color of the SBs of our sample. The different symbols identify the compactness class (Hattori et al. 2004), while the label shows the morphological type.

Table 7. Input parameters for the reference simulation.

\begin{tabular}{|c|c|}
\hline$L_{\mathrm{TIR}} / \mathrm{SFR}$ calibration & Kennicutt (1998) \\
\hline IMF & Salpeter \\
\hline SFR distribution & $\begin{array}{l}\propto L_{K}^{\alpha} \\
\text { for compactness class } 1,2,3,4 \\
\alpha=2.0,1.7,1.25,1.0\end{array}$ \\
\hline$A_{V}$ & $\propto \mathrm{SFR}(\max 30 \mathrm{mag})$ \\
\hline $\begin{array}{l}\text { CC mass range } \\
\text { Ia components }\end{array}$ & $\begin{array}{l}8-50 M_{\odot} \\
30 \% \text { prompty, } 70 \% \text { tardy }\end{array}$ \\
\hline SN luminosity function & LOSS (Li et al. 2011b) \\
\hline
\end{tabular}

SBs with strong tidal features and a single nucleus were classified as "mergers" (M), galaxy pairs with an overlapping disk or a connecting bridge were classified as "close pairs" (CP) if the projected separation is $<20 \mathrm{Kpc}$ and galaxies that have a nearby $(<100 \mathrm{Kpc})$ companion at the same redshift were classified as "pairs" (P). The remaining objects were classified as "single" (S). The classification of the SBs of our sample is listed in Table 6 along with the galaxy FIR colors, $\log f_{60} / f_{100}$, $\log f_{25} / f_{60}$.

We attributed to each galaxy a compactness class on the basis of its correlation with the FIR colors as shown in Fig. 4 of Hattori et al. (2004), which for the object of our sample corresponds to our Fig. 8. As can be seen, we also confirmed their claim of a (weak) relation of FIR color and, as a consequence, compactness class with SB morphology.

The next step is based on Soifer et al. (2000, 2001). For several SBs galaxies they plotted the MIR and NIR emission curve of growth finding that, in general, the MIR emission is more concentrated, while only for a few galaxies do the MIR and NIR curves of growth show a similar trend. Actually we found that, to a first-order approximation, the MIR emission profile of a given galaxy can be matched by NIR profile powered to an exponent $\alpha$, which ranges between 1 , when the two profiles are similar, to 2, when the MIR emission is strongly concentrated. When we classified the same galaxies with the compactness criteria of Hattori et al. (2004), we find that (as expected) the galaxies with compact SF regions (types 1-2) are characterized by more concentrated MIR emission ( $\alpha=2.0-1.5$, respectively), while galaxies with extended SF region (types 3-4) have similar MIR vs. NIR profiles ( $\alpha=1.25-1.0$, respectively). As a reference, we notice that in the typical case of NGC 6240, assuming $\alpha=1$ corresponds to locate $50 \%$ of the SFR within $1.5 \mathrm{Kpc}$, whereas for $\alpha=2$ the same SFR fraction is enclosed within $500 \mathrm{pc}$.

As a result of this discussion we estimate the SF distribution based on the observed $L_{K}$, and adopt a power index $\alpha$ appropriate to the compactness class of the given SB galaxy (Table 7).

\subsubsection{Flow chart of the simulation}

Having defined all the ingredients of the simulation, we can now describe how this proceeds. The simulation flowchart can be summarized as follows:

1. For each galaxy of the sample, based on the estimated total SFR and adopted progenitor scenarios, we compute the expected number of $\mathrm{SNe}$ per year.

2. A time interval is chosen so that $100 \mathrm{SNe}$ are expected to explode in the given galaxy in that period. The time interval ends with the last observations of the galaxy. Given the expected $\mathrm{SN}$ rates, it is much longer for all galaxies than the duration of our monitoring campaign. The reason for simulating 100 events is to avoid having to deal with fractional SN numbers for the different subtypes. We assign to each event a random epoch of explosion chosen within the defined time interval.

3. Each $\mathrm{SN}$ is assigned to a random explosion site inside the parent galaxy according to the SFR spatial distribution.

4. A peak magnitude is also assigned to each $\mathrm{SN}$, with a random value derived from the adopted SN luminosity function for the specific subtype. We also associate an extinction value to the event that is randomly extracted from a Gaussian distribution whose mean value depends on the position of the SN, and $\sigma=1 / 3$ of the mean value.

5. The apparent magnitude is thereafter calculated at the epochs of available observations, knowing the galaxy distance modulus and SN epoch of explosion. This is compared with the search's magnitude detection limit for the given position, and when brighter, the $\mathrm{SN}$ is added to the simulated discovery list.

The process, iterated for all the galaxies of the sample, defines a single simulation run. Outcomes of the simulation are the expected number of SN discoveries, their types, magnitudes, extinctions, and positions inside the host galaxies. To explore the distribution of the outcomes from the random process, a complete experiment is made by collecting a minimum of a hundred single simulation runs.

\section{Comparison between observed and expected SN discoveries}

As we outlined above, from many of MonteCarlo simulation runs we obtain the distribution of the expected SN discoveries. This is shown in Fig. 9, where each bin of the histogram is the predicted probability of observing the specific number of SN discoveries, whereas the dashed line marks the number of actual $\mathrm{SNe}$ discovered and the shaded area shows its 1- $\sigma$ Poissonian uncertainty range.

We found that with the adopted simulation scenario and input parameters, we should have expected, on average, to discover 


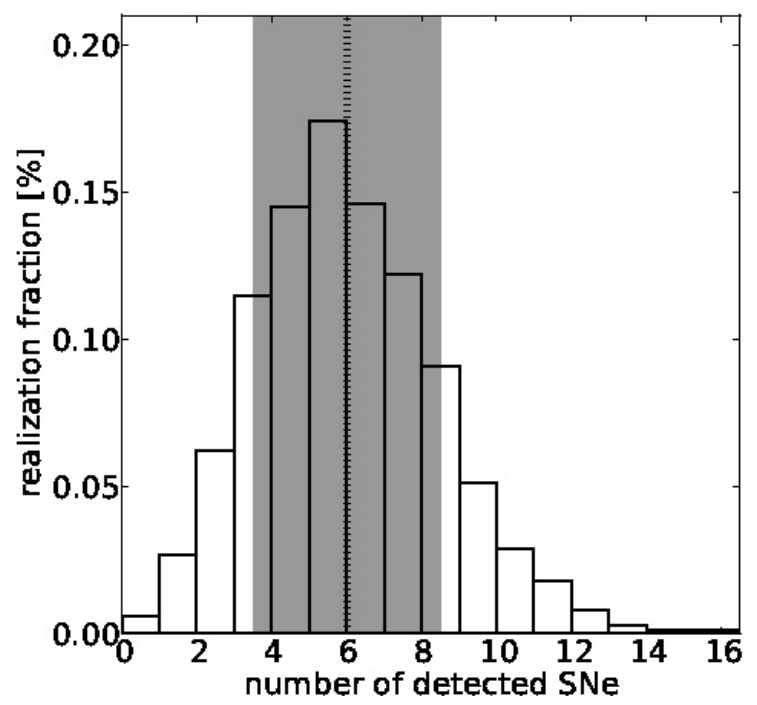

Fig. 9. Histogram of the number of expected SNe from our MonteCarlo experiments. The dashed vertical line indicates the number of observed events and the gray area its $1 \sigma$-Poissonian uncertainty.

$5.3 \pm 2.3 \mathrm{SNe}$. In $68 \%$ of the experiments $(1-\sigma)$ the expected number is in the range $4-8$, which is in excellent agreement with the observed number of six events. The prediction of the simulation is that almost all $\mathrm{SNe}$ are CC (5.1 SN CC vs. 0.2 SN Ia), though in $10 \%$ of the experiments at least one type Ia is found (that is what we have from the real SN search).

The distributions of some of the expected and observed SN properties are compared in Fig. 10. For the simulation, we show the distribution across the different experiments (line-only histogram) while the grey shaded histogram represents the actual observations.

The top panel in Fig. 10 shows the distribution of the apparent magnitudes at the discovery. The good agreement between simulations and observations is a crucial consistency check of our estimates of the magnitude detection limit: if the discovered SNe were systematically fainter/brighter then expected, this would indicate an underestimate/overestimate of the search detection efficiency.

A comparison of the simulated vs. observed extinction distribution is shown in the middle panel of Fig. 10. For the observed distribution, the case of PSN2010 is shown for which extinction is ambiguous is shown. Again the simulation is in good agreement with the observations. This argues in favor of the consistency of the input assumptions. The fact that, in our IR search, we expect that most detected $\mathrm{SNe}$ have low extinction $(\sim 75 \%$ with $A_{V}<1 \mathrm{mag}$ ) is a consequence of our assumption that the extinction is very high in the nucleus and rapidly decreases with the galaxy radius, following the same trend in the SFR. This does not mean that extinguished $\mathrm{SN}$ are intrinsically rare, but that they are confined to the galaxy nuclear regions where extinction is extremely high even in the IR (see next). At the same time we can exclude the presence of any significant population of $\mathrm{SNe}$ with intermediate extinctions: we would easily detect them in our infrared search. Mattila \& Meikle (2001) find an average value of $A_{V}=30 \mathrm{mag}$ for the extinction towards the SN remnants of M82, confirming the presence of high extinction (about $A_{V} \sim 15-45 \mathrm{mag}$ ) in the innermost $300 \mathrm{pc}$ regions. On the other hand, Kankare et al. (2008) find an host galaxy extinction of $A_{V} \sim 16$ mag for the SN $2008 \mathrm{cs}$, located at about $1.5 \mathrm{Kpc}$, so relatively far from the galaxy nucleus.

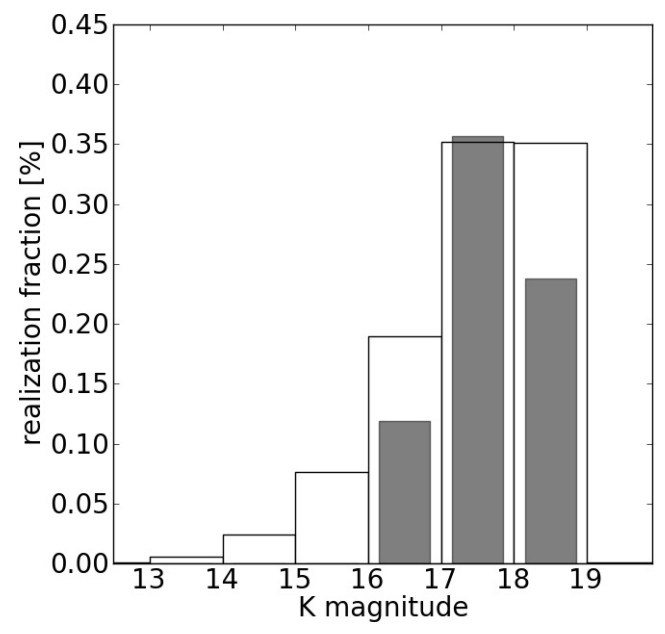

(a)

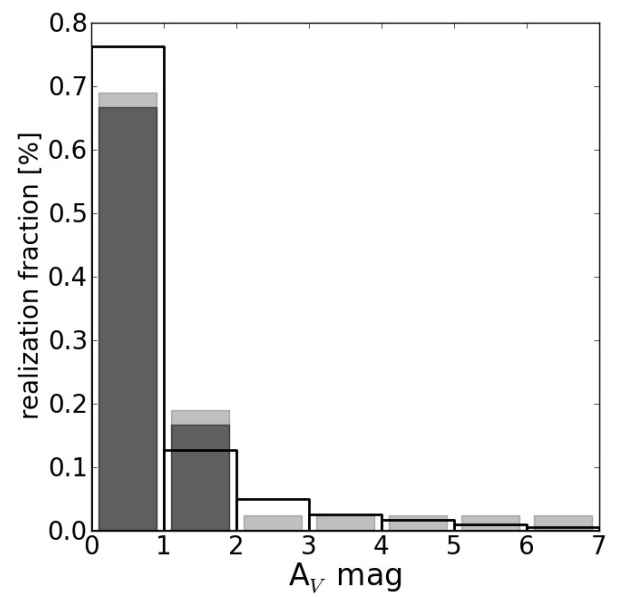

(b)

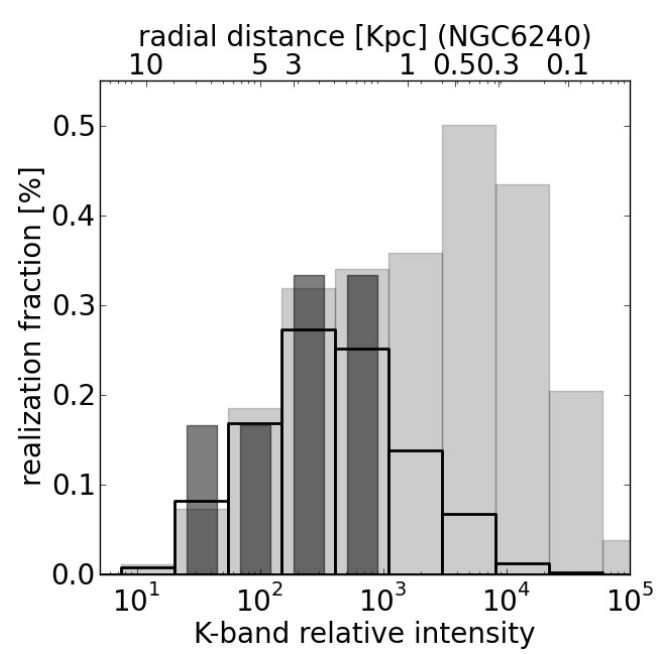

(c)

Fig. 10. SN property comparison. a) Expected (line-only) and observed (gray) $K$ magnitude distribution at the discovery. b) Expected (lineonly) and observed (gray) extinction distribution. In light gray we indicate the allowed range for the extinction of PSN2010 (see text). c) Radial distribution of expected (line-only) and detected (dark grey) $\mathrm{SNe}$. For regular galaxies the surface brightness decreases monotonically with radial distance. The upper axis shows this correspondence for one of the galaxy of our sample. In light gray we show the distribution of injected artificial SNe (see text). 
Finally, in the bottom panel of Fig. 10 we compare the distribution of locations inside the host galaxy for the expected vs. observed SNe. The different location are identified by the $K$ band pixel counts: in general high counts occur in the nuclear regions, while low counts are in the outskirts. We use pixel counts instead of radial distances because the latter is difficult to be defined for galaxies with irregular morphology or double nuclei. However an indicative correspondence from pixel count to radial distance is shown at the top axis of the figure for a galaxy with regular morphology. There is a mild indication of a deficiency of observed events in regions with high pixel counts. Taken at face value this may suggest a minor overestimate of the detection magnitude limit in the nuclear regions. Given the lack of statistics we cannot raw definite conclusions and therefore will not develope this issue further.

In the same figure we also show the distribution of locations of the expected events for an ideal case where the magnitude detection limit in the nuclear regions is as deep as in the outskirts and extinction is negligible. The experiment shows that the fraction of events that remain hidden to our search in the galaxy nuclear regions due to the combined effect of reduced search efficiency, and extinction is very high, about $60 \%$ (cf. Mattila et al. 2012).

\subsection{Uncertainties and effect of different assumptions}

\section{Magnitude detection limit}

One of the main sources of uncertainty for the simulation is related to the estimate of the magnitude detection limit, $\mathrm{mag}_{\mathrm{lim}}$. For the reference simulation, we adopted the mean value as $m a g_{\text {lim }}$ out of three artificial star experiments conducted for several selected positions inside the host galaxy (cf. 2.5). The dispersion of measurements, which is the uncertainty on $\mathrm{mag}_{\mathrm{lim}}$, is quite large with a typical range of $\sim 0.5 \mathrm{mag}$, but in more extreme difficult cases, it can be as large as 2 mag.

To test the propagation of this uncertainty, we performed MonteCarlo experiments by alternatively assuming the lower and higher $\mathrm{mag}_{\text {lim }}$ out of the three experiments. We found that the predicted number of SNe is $6.2 \pm 2.5$ and $4.7 \pm 2.2$, which are $+17 \%$ and $-11 \%$ with respect to the numbers from the reference simulation. That error is significant is why we made a significant effort to get a detailed estimate of the detection limit.

\section{SN luminosity function}

In the reference simulation we use a Gaussian distribution for the SN luminosity function (SN-LF) with a mean value and dispersion taken from Li et al. (2011b). However, based on a small sample of very nearby SNe, Horiuchi et al. (2011) claim that the faint end of the SN-LF is underestimated, and SN CC fainter than mag $\simeq-16$ could make up to $50 \%$ of the distribution, to be compar with $20 \%$ of the sample of Li et al. (2011b). On the other hand, Mattila et al. (2012) argue that Horiuchi et al. (2011) overestimated the fraction of intrisically faint CCSNe since they neglect the host galaxy extinction for their SN absolute magnitudes. We performed a MonteCarlo experiment that adopt the Horiuchi's SN-LF and found that in this case the expected number of events would be low, only 3.3 on average. This is because most faint events are expected to fall below the search detection limit. That the actual discoveries are twice this number argues against a large fraction of faint SN-CC (cf. Botticella et al. 2012).

\section{IMF and SN CC progenitor mass range}

The IMF enters both in the estimate of the number of SN progenitors and in the calibration of TIR luminosity in terms of SFR relation. However, the expected rate of CC SNe in our sample is virtually independent of the IMF slope. Indeed, for a given total mass of the parent stellar population, top heavy IMFs imply both more of CC progenitors and higher luminosity. Following Dwek et al. (2011), the number of CC progenitors per unit mass is $k_{\mathrm{CC}}=0.007,0.011$, and $0.039 M_{\odot}{ }^{-1}$ for a Salpeter, a Kroupa, and a Starburst IMF respectively, assuming that the progenitors range from 8 to $50 M_{\odot}$. At the same time, the total luminosity of an SB forming stars with an SFR of $1 M_{\odot} \mathrm{yr}^{-1}$ over a period of $10 \mathrm{Myr}$ (i.e. a $10^{7} M_{\odot}$ stellar population) is $4.71 \times 10^{9}, 7.33 \times 10^{9}$, and $2.55 \times 10^{10} L_{\odot}$ again for a Salpeter, a Kroupa, and a Starburst IMF. The M/L ratio of such SBs is then 0.0021, 0.0014, and 0.0004 (solar units) for the three IMFs, and the expected number of CC SNe originating from it is $\simeq 1.5$ every $10^{5} \mathrm{~L}_{\odot}^{-1}$ for all three IMFs. Working out the numbers, it turns out that the SN CC rates from a population with a given $L_{\mathrm{TIR}}$ is almost independent of the IMF, provided a consistent choice is made.

Crucial is instead the assumption of the SN CC progenitor mass range, in particular, the lower limit. Indeed if we adopted an upper limit of $100 M_{\odot}$ instead of the reference value of $50 M_{\odot}$, the expected number of SNe would be $5.5 \pm 2.1$, only $\sim 5 \%$ higher then the reference simulation. On the other hand, assuming a lower limit of $10 M_{\odot}$ (instead of $8 M_{\odot}$ ) results in an expected number of SNe of $3.9 \pm 2.1$, which is $\sim 30 \%$ lower than the expected rate obtained in the reference case.

\section{Extinction}

For the reference case we assumed that the extinction scales with the SFR with a maximum value corresponding to the SFR peak $A_{V}=30 \mathrm{mag}$. To test the uncertainty related to this assumption, we made two different tests. In one experiment we maintained the relation of $A_{V}$ with SFR but taking, alternatively, peak extinction values $A_{V}=10$ and $A_{V}=100 \mathrm{mag}$. The experiment gave $5.5 \pm 2.7$ and $4.7 \pm 2.3$ respectively as expected number of $\mathrm{SNe}$. In the second experiment we assumed that the extinction is constant through the galaxy and is $A_{V}=3.0 \mathrm{mag}$. In this case the expected number is $5.3 \pm 2.3$, so identical to the value of the reference simulation.

The conclusion is that the uncertainty on the extinction does not significantly affect the simulation or, conversely, that our experiment cannot probe the extinction distribution.

\section{Star formation distribution}

The spatial distribution of SFR is an important, and the most uncertain, ingredient of the simulation. For instance, if we assume that the SFR is confined in the very inner regions, say in the inner 3-500 pc, the resulting SNe will remain inaccessible to our search. On the other hand, that the SFR is extended in some SBs has been confirmed by different studies (eg. McQuinn et al. 2012), not to mention that many of the SNe we have discovered are at significant radial distances (cf. Table 3).

As described in Sect. 3.1.4, as proxy of the SFR distribution we use $L_{K}^{\alpha}$ where $\alpha$ range from 1 to 2 depending on the galaxy compactness class (Table 6). To test for the uncertainties of this assumption we performed two simulations that assume that $\alpha$ is either 1 or 2 for all galaxies. We obtained an expected rate of $8.8 \pm 3.0$ in the first case and in the second case a value of $3.0 \pm 1.7$. The latter occurs because when the SFR is more 
concentrated, a large number of $\mathrm{SNe}$ remain hidden to our search due to the low search detection efficiency in the nuclear regions.

The conclusion is that the uncertainty in the adopted SF distribution propagates with an error of $\sim 50 \%$ on the expected SN number. We may consider that the actual good match of observations with the reference simulation argues in favor of the adopted prescription.

\section{Summary and conclusions}

We have presented the analysis of an infrared SN search in a sample of 30 nearby SB galaxies, conducted between 2009 and 2011, with the goal verifying the link between star formation and the SN rate. During our search we collected about 240 observations in total, discovering $6 \mathrm{SNe}, 4$ of them with spectroscopic confirmation.

To verify how this number compare with expactations we performed a detailed characterization of the SN detection efficiency, the galaxy properties (in particular SF rate and spatial distribution), and the SN properties and progenitor scenarios. We included all these ingredients in a MonteCarlo simulation tool that, after allowing for the stochastic nature of SN events, can be used to explore the distribution of the expected SN number and properties.

First of all, we may point out that by itself the number of detected SNe is proof of the high SFR in SBs. In fact if we computed the expected number of SNe in our survey based on the average $\mathrm{SN}$ rate per unit $B$ luminosity or mass (Li et al. 2011a), we would predict the discovery of 0.5 events (or more precisely, $50 \%$ of the simulation predict the discovery of one event and none is expected in the other $50 \%$ ). The observed number is one order of magnitude higher, which is consistent with the fact that the TIR emission of SBs is about ten times higher than for normal SF galaxies with the same B luminosity. Indeed, it is well known that the TIR luminosity is an excellent tracer for SFR, in particular in SBs.

When we adopted the SFR from $L_{\mathrm{TIR}}$ as input for the MonteCarlo experiment, we found that the expected number of $\mathrm{SNe}$ in our search is $5.3 \pm 2.3, \mathrm{SNe}$ in excellent agreement with observations.

We performed a number of tests to verify the dependence of the simulation outcomes from the input parameters. For the SN search characterization we show that an accurate estimate of the magnitude limit for SN detection is crucial. This is why we made a considerable effort in artificial star experiments (possibly the single most expensive task of our project). For the galaxy characterization, the most uncertain input is the SF spatial distribution. With some creativity, we devised a prescription that seems to work, but it is certain that this is a place for improvements when new, high-resolution SB maps become available. Instead, we found that our results are not sensitive to the uncertainty on the amount of extinction because where extinction is very high (the dense SB regions) our search is limited by the bright magnitude detection limit. $\mathrm{SNe}$ in these regions remain hidden to our search almost independently of the amount of extinction. Based on our simulation we estimated that the fraction of hidden $\mathrm{SNe}$ is very significant, that is, $\sim 60 \%$ with an upper limit of $75 \%$, if we account for the Poissonian uncertainties in the number of detected events. Finally, for the SN progenitor scenarios the larger uncertainty is the lower limit of the progenitor mass range. If we adopt a lower limit $M_{\mathrm{CC}}^{\mathrm{L}}=10 M_{\odot}$ instead of $8 M_{\odot}$ as in the reference simulation, the expected number of SNe would be $30 \%$ lower than observed.
Our results appear to agree with those of previous, similar searches (Mannucci et al. 2003; Cresci et al. 2007; Mattila et al. 2007a, 2012, cf. Sect. 1). In broad terms, the overall conclusion of all these studies can be expressed as follows. The number of (CC) SNe found in SBs galaxies is consistent with what is predicted from the high SFR (and the canonical mass range for the progenitors) when we recognize that a major fraction of the events remain hidden in the inaccessible SB regions. As stressed by Mattila et al. (2012), this has important consequences for the use of SN CC as a probe of the cosmic SFR, because the fraction of SBs is expected to increase with redshifts (cf. Melinder et al. 2012; Dahlen et al. 2012)

While continuing to search for $\mathrm{SNe}$ in $\mathrm{SBs}$, in the optical, and infrared, it can certainly help to improve the still lack of statistics, one may argue at this point for a change in strategy.

In this respect a good example is the attempt to reveal some of the hidden SN CC through infrared SN searches that exploit adaptive optics at large telescopes, e.g. Gemini or VLT. The results are encouraging with the discovery of two $\mathrm{SNe}$ with very high extinction, namely SN 2004ip with $A_{V}$ between 5 and 40 mag (Mattila et al. 2007b) and SN 2008cs with $A_{V} 16$ mag (Kankare et al. 2008), though we may notice that both objects were too faint for spectroscopic confirmation. Other two $\mathrm{SNe}$ were discovered very close to the galaxy nucleus, namely SN 2010cu at a radial distance of $180 \mathrm{pc}$ and SN 2011 hi at $380 \mathrm{pc}$ (Kankare et al. 2012), though in these cases the low extinction suggests that the low radial distance is a projection effect. Also in these cases no spectroscopic classification was obtained. The extinction towards SN 2011hi was revised by Romero-Cañizales et al. (2012) using Gemini-N data. They demonstrate that this is most likely an SN IIP with $A_{V}$ of 5-7 mag. Because of the need to monitor one galaxy at a time and to access heavily subscribed large telescopes, this approach will not result in large statistical sample though even a few events may be mostly valuable for exploring the very obscured nuclear regions.

On the other hand, a new opportunity that should be explored is the piggy-back-on-wide-field extragalactic surveys of the next generation of infrared facilities, in particular EUCLID. This would allow IR SN searches to be performed for the first time on a large sample of galaxies exploring a range of SF activity and, by monitoring galaxies at different redshifts, probe the cosmic evolution.

Acknowledgements. We thank the referee, Seppo Mattila, for the careful reading and the very useful comments. We give particular thanks to Anna Feltre (ESO) for her help in estimating the possible contribution by AGNs to the FIR luminosity of the galaxies and to Barbara Lo Faro (Astronomy Department of Padova) for helpful discussions and suggestions. We acknowledge the support of the PRIN-INAF 2009 with the project "Supernovae Variety and Nucleosynthesis Yields". E.C., L.G., S.B., A.P., and M.T. are partially supported by the PRIN-INAF 2011 with the project "Transient Universe: from ESO Large to PESSTO”. N.E.R. acknowledges financial support by the MICINN grant AYA08-1839/ESP, AYA2011-24704/ESP, and by the ESF EUROCORES Program EUROGENESIS (MINECO grants EUI2009-04170). F.B. acknowledges support from FONDECYT through Postdoctoral grant 3120227 and from the Millennium Center for Supernova Science through grant P10-064-F (funded by "Programa Bicentenario de Ciencia y Tecnologia de CONICYT" and "Programa Iniciativa Cientifica Milenio de MIDEPLAN").

\section{References}

Alard, C. 2000, A\&AS, 144, 363

Bazin, G., Palanque-Delabrouille, N., Rich, J., et al. 2009, A\&A, 499, 653

Benetti, S., Turatto, M., Cappellaro, E., Danziger, I. J., \& Mazzali, P. A. 1999. MNRAS, 305, 811

Bertin, E., \& Arnouts, S. 1996, A\&AS, 117, 393

Blanc, G., \& Greggio, L. 2008, New Astron., 13, 606

Botticella, M. T., Riello, M., Cappellaro, E., et al. 2008, A\&A, 479, 49 
Botticella, M. T., Smartt, S. J., Kennicutt, R. C., et al. 2012, A\&A, 537, A132 Bregman, J. D., Temi, P., \& Rank, D. 2000, A\&A, 355, 525 Calzetti, D., Armus, L., Bohlin, R. C., et al. 2000, ApJ, 533, 682

Cappellaro, E., Turatto, M., Tsvetkov, D. Y., et al. 1997, A\&A, 322, 431 Cappellaro, E., Evans, R., \& Turatto, M. 1999, A\&A, 351, 459 Cappellaro, E., Riello, M., Altavilla, G., et al. 2005, A\&A, 430, 83

Cresci, G., Mannucci, F., Della Valle, M., \& Maiolino, R. 2007, A\&A, 462, 927

Dahlen, T., Strolger, L.-G., Riess, A. G., et al. 2004, ApJ, 613, 189

Dahlen, T., Strolger, L.-G., Riess, A. G., et al. 2012, ApJ, 757, 70

Dale, D. A., Helou, G., Contursi, A., Silbermann, N. A., \& Kolhatkar, S. 2001, ApJ, 549, 215

Dwek, E., Staguhn, J. G., Arendt, R. G., et al. 2011, ApJ, 738, 36

Elmhamdi, A., Danziger, I. J., Chugai, N., et al. 2003, MNRAS, 338, 939

Engel, H., Davies, R. I., Genzel, R., et al. 2011, ApJ, 729, 58

Folatelli, G., Gutierrez, C., \& Pignata, G. 2010, Central Bureau Electronic Telegrams, 2390, 1

Gallagher, III, J. S. 1993, in Massive Stars: Their Lives in the Interstellar

Medium, eds. J. P. Cassinelli, \& E. B. Churchwell, ASP Conf. Ser., 35, 463

Goldoni, P., Royer, F., François, P., et al. 2006, in SPIE Conf. Ser., 6269

Graur, O., Poznanski, D., Maoz, D., et al. 2011, MNRAS, 417, 916

Greggio, L. 2005, A\&A, 441, 1055

Greggio, L. 2010, MNRAS, 406, 22

Greggio, L., \& Renzini, A. 1983, A\&A, 118, 217

Grossan, B., Spillar, E., Tripp, R., et al. 1999, AJ, 118, 705

Harutyunyan, A. H., Pfahler, P., Pastorello, A., et al. 2008, A\&A, 488, 383

Hattori, T., Yoshida, M., Ohtani, H., et al. 2004, AJ, 127, 736

Helou, G., Khan, I. R., Malek, L., \& Boehmer, L. 1988, ApJS, 68, 151

Hopkins, A. M., \& Beacom, J. F. 2006, ApJ, 651, 142

Horiuchi, S., Beacom, J. F., Kochanek, C. S., et al. 2011, ApJ, 738, 154

Hunter, D. J., Valenti, S., Kotak, R., et al. 2009, A\&A, 508, 371

Inami, H., Armus, L., Surace, J. A., et al. 2010, AJ, 140, 63

Kankare, E., Mattila, S., Ryder, S., et al. 2008, ApJ, 689, L97

Kankare, E., Mattila, S., Ryder, S., et al. 2012, ApJ, 744, L19

Kennicutt, R. C., Jr. 1998, ApJ, 498, 541

Kennicutt, R. C., \& Evans, N. J. 2012, ARA\&A, 50, 531

Li, W., Chornock, R., Leaman, J., et al. 2011a, MNRAS, 412, 1473

Li, W., Leaman, J., Chornock, R., et al. 2011b, MNRAS, 412, 1441

Maiolino, R., Vanzi, L., Mannucci, F., et al. 2002, A\&A, 389, 84

Mannucci, F., Maiolino, R., Cresci, G., et al. 2003, A\&A, 401, 519

Mannucci, F., Della Valle, M., Panagia, N., et al. 2005, A\&A, 433, 807
Mannucci, F., Della Valle, M., \& Panagia, N. 2006, MNRAS, 370, 773

Mannucci, F., Della Valle, M., \& Panagia, N. 2007, MNRAS, 377, 1229

Maoz, D., \& Mannucci, F. 2012, PASA, 29, 447

Maoz, D., Mannucci, F., \& Brandt, T. D. 2012, MNRAS, 426, 3282

Marion, G. H., Challis, P., \& Berlind, P. 2010, Central Bureau Electronic Telegrams, 2449, 1

Mattila, S., \& Meikle, W. P. S. 2001, MNRAS, 324, 325

Mattila, S., Meikle, P., Greimel, R., \& Väisänen, P. 2007a, in IAU Symp. 235, eds. F. Combes, \& J. Palouš, 323

Mattila, S., Väisänen, P., Farrah, D., et al. 2007b, ApJ, 659, L9

Mattila, S., Dahlen, T., Efstathiou, A., et al. 2012, ApJ, 756, 111

Maza, J., Hamuy, M., Antezana, R., et al. 2010, Central Bureau Electronic Telegrams, 2388, 1

McQuinn, K. B. W., Skillman, E. D., Cannon, J. M., et al. 2009, ApJ, 695, 561

McQuinn, K. B. W., Skillman, E. D., Dalcanton, J. J., et al. 2012, ApJ, 759, 77

Melinder, J., Dahlen, T., Mencía Trinchant, L., et al. 2012, A\&A, 545, A96

Miluzio, M., \& Cappellaro, E. 2010, Central Bureau Electronic Telegrams, 2446, 1

Miluzio, M., Benetti, S., Botticella, M. T., et al. 2011, Central Bureau Electronic Telegrams, 2773, 1

Monard, L. A. G. 2010, Central Bureau Electronic Telegrams, 2250, 1

Navasardyan, H., Petrosian, A. R., Turatto, M., Cappellaro, E., \& Boulesteix, J. 2001, MNRAS, 328, 1181

Pastorello, A., Valenti, S., Zampieri, L., et al. 2009, MNRAS, 394, 2266

Paturel, G., Petit, C., Prugniel, P., et al. 2003, A\&A, 412, 45

Richmond, M. W., Filippenko, A. V., \& Galisky, J. 1998, PASP, 110, 553

Romero-Cañizales, C., Pérez-Torres, M. A., Alberdi, A., et al. 2012, A\&A, 543, A72

Sanders, D. B., \& Mirabel, I. F. 1996, ARA\&A, 34, 749

Sanders, D. B., Mazzarella, J. M., Kim, D.-C., Surace, J. A., \& Soifer, B. T. 2003, AJ, 126, 1607

Scannapieco, E., \& Bildsten, L. 2005, ApJ, 629, L85

Shioya, Y., Trentham, N., \& Taniguchi, Y. 2001, ApJ, 548, L29

Smartt, S. J. 2009, ARA\&A, 47, 63

Soifer, B. T., Neugebauer, G., Matthews, K., et al. 2000, AJ, 119, 509

Soifer, B. T., Neugebauer, G., Matthews, K., et al. 2001, AJ, 122, 1213

Totani, T., Morokuma, T., Oda, T., Doi, M., \& Yasuda, N. 2008, PASJ, 60, 1327

Turatto, M., Benetti, S., Cappellaro, E., \& Bufano, F. 2010, Central Bureau Electronic Telegrams, 2252, 1 